\title{
Immuno-activated mescenchymal stem cell living electrospun nanofibers for promoting diabetic wound repair
}

\section{Shaoying Gao}

Affiliated hospital of Zunyi Medical University

\section{Tao Chen}

Affiliated hospital of Zunyi Medical University

\section{Zhen Wang}

Ruijin Hospital, Shanghai Jiao Tong University School of Medicine

\section{Ping Ji}

Shanghai Jiao Tong University School of Medicine

\section{Lin Xu}

Zunyi Medical University

\section{Wenguo Cui}

Ruijin Hospital, Shanghai Jiao Tong University School of Medicine

\section{Ying Wang ( $\nabla$ ywangssmu@shsmu.edu.cn )}

Shanghai Jiao Tong University School of Medicine

\section{Method Article}

Keywords: Diabetic wound healing, M1-like macrophages, cell membrane, electrospun nanofibers, bone marrow-derived mesenchymal stem cells

Posted Date: March 8th, 2022

DOI: https://doi.org/10.21203/rs.3.rs-1403530/v1

License: (c) (1) This work is licensed under a Creative Commons Attribution 4.0 International License. Read Full License 
Immuno-activated mescenchymal stem cell living electrospun nanofibers for promoting diabetic wound repair

Shaoying $\mathrm{Gao}^{\mathrm{a}, \mathrm{b}}$, Tao Chen ${ }^{\mathrm{a}}$, Zhen Wang ${ }^{\mathrm{c}}$, Ping $\mathrm{Ji}^{\mathrm{b}}$, Lin $\mathrm{Xu}^{\mathrm{d}^{*}}$, Wenguo Cui ${ }^{*}$, Ying Wang $^{b^{*}}$

a. Department of Burn plastic surgery, Affiliated hospital of Zunyi Medical University, Guizhou, 563000, China

b. Shanghai Institute of Immunology, Department of Immunology and Microbiology, Key Laboratory of Cell Differentiation and Apoptosis of Chinese Ministry of Education, Shanghai Jiao Tong University School of Medicine, Shanghai, 200025, China

c. Shanghai Key Laboratory for Prevention and Treatment of Bone and Joint Diseases, Shanghai Institute of Traumatology and Orthopaedics, Ruijin Hospital, Shanghai Jiao Tong University School of Medicine, 197 Ruijin Second Road, Shanghai, 200025, PR China.

d. Department of Immunology, Special Key Laboratory of Gene Detection and Therapy \& Base for Talents in Biotherapy of Guizhou Province, Zunyi 563000, China

Corresponding authors: Ying Wang, PhD. Email: ywangssmu@shsmu.edu.cn Wenguo Cui, PhD. Email: wgcui80@hotmail.com Lin Xu, PhD. Email: xulinzhouya@163.com

Shaoying Gao and Tao Chen contributed equally to this work. 


\section{Abstract}

Diabetic wound is the leading cause of non-traumatic amputations in which oxidative stress and chronic inflammation are main factors affecting wound healing. Although conventional mesenchymal stem cells (MSCs) living material can promote skin regeneration, they are still vulnerable to oxidative stress which limits their clinical applications. Here, we have prepared (lactic-co-glycolic acid) (PLGA) nanofibers electrospun with LPS/IFN- $\gamma$ activated macrophage cell membrane with the capacity to immunostimulate bone marrow derived mesenchymal stem cells (BMMSCs) and investigated the effects of this living material on diabetic wound healing. After defining the physic-chemical properties of LPS/IFN- $\gamma$ activated RAW264.7 cell(a mouse M1 macrophage cell line model) membrane modified nanofibers (RCM-fibers), including surface molecule, diameter, hydrophilicity and degradation rate, we demonstrated that the RCM-fibers not only improved BMMSC proliferation and keratinocyte migration upon oxidative stress in vitro, but also accelerated BMMSCs-mediated wound closure with rapid re-epithelialization, collagen remodeling, immunoregulation, antioxidant stress and angiogenesis in experimental diabetic wound healing in vivo. Transcriptome analysis revealed the up-regulation of genes related to wound healing in BMMSCs upon RCM-fiber immune-stimulation. Enhanced healing capacity of RCM-fiber-BMMSCs living material was partially mediated through CD200-CD200R interaction. Similarly, LPS/IFN- $\gamma$ activated THP-1 cell(a human M1 macrophage cell line model) membrane coated nanofibers (TCM-fibers) exhibited the similar improvement of human BMMSCs (hBMMSCs) on the diabetic wound healing in vivo. Our results thus demonstrate that LPS/IFN- $\gamma$ activated macrophage cell membrane-modified nanofibers can in situ promote the biofunction of BMMSCs, making this novel living material promising in wound repair for human diabetes.

Key words: Diabetic wound healing, M1-like macrophages, cell membrane, 
electrospun nanofibers, bone marrow-derived mesenchymal stem cells

\section{Introduction}

Conventional biomaterials-based platform, like nanofibers or hydrogels, provides biophysical support as well as integrates bioactive molecules to instruct biological functionality in tissue regeneration ${ }^{[1]}$. Although these biomaterials have been successfully applied in pre-clinical investigations, considering our tissues are composed of living cells and cell-secreted extracellular matrices (ECMs) capable of adapting to diverse biological scenarios, living materials have been conceptualized and further engineered. These engineered living materials possess multiple advantages including capable of generating biologics responding to local environment and yielding more adaptations through autonomously biological behaviors $^{[2]}$. At present, bacteria, fungi and stem cells have been used in living materials for a range of applications such as biosensing, tissue regeneration and drug delivery $[3,4]$. Living materials exhibit "living character" through living cells on polymeric matrice blocks constructing bioactive and bioresponsive units ${ }^{[5]}$. Until now, living materials containing mesenchymal stem cells (MSC), keratinocytes or fibroblasts have been highly recommended in wound care and wound management lies in the fact that they are either demonstrated to ameliorate wound healing processes (like MSCs) or main components in the wound regions to be reprogrammed during wound healing ${ }^{[6]}$. Polymeric matrices of living materials are likely to improve regenerative performances over conventional biomaterials partially through providing more cell adhesion capability, maintaining the merits and characteristics of MSCs, as well as promoting cell survival, proliferation, and differentiation through mimicking the in vivo microenvironment via cell-matrix interactions ${ }^{[7]}$.

Diabetes associated nonhealing chronic wounds have become one of the main common complications among non-traumatic amputations nowadays. Although wound care management is well documented including haemostasis, antiinflammation, cell proliferation and remodeling $\left.{ }^{[8,} 9\right]$, diabetic wounds is still refractory to currently available treatment for chronic wounds owing to several 
unsolved difficulties including increased regional oxidative stress, dysfunctionality of immune cells and keratinocytes as well as impaired angiogenesis etc ${ }^{[10]}$. In addition, high glucose and subsequent oxidative stress in wound regions of diabetic patients also cause severe damage to the cells of living materials. Considering the alteration of internal environment suffering from chronic diseases such as diabetes, the cells of living materials might be affected and the living materials may not execute normal function. The design of living materials according to pathologic circumstance for wound healing still needs inspiration.

Among nanofiber living materials, nanofiber scaffolds provide a solid matrix for cell attachment and proliferation, and supply physicochemical and/or bioactive cues to the resident cells ${ }^{[11]}$. The interactions between the cells and the scaffolds are of particular importance as these interactions determine the survival and function of the cells ${ }^{[12]}$. Therefore, scaffolds, typically made of polymeric biomaterials such as polymeric nanofibers, are often modified with cell surface receptors for intracellular signaling, which may alter protein expression and modulate cell function of the resident cells ${ }^{[13]}$. The lipid membranes not only shield the nanofibers from the external environment but also provide a biomolecule-friendly medium in which to anchor cell surface receptors and preserve their integrity and functionality ${ }^{[14]}$. Although these bottom-up functionalization approaches are sufficient to present individual receptors or receptor combinations to the resident cells in the scaffolds, they are generally inadequate for recapitulating the complexity and functions of cell surface which are critical for cell-cell interaction and cell functions in the scaffolds ${ }^{[15]}$. Therefore, cell membranes derived from living cells are coating directly on polymeric nanofibers, which becomes a new and robust strategy in nanofiber scaffold modification to improve the biofunctionality of living materials.

The use of cell membranes to cloak synthetic nanoparticles through a top-down fabrication method has emerged as a promising technique for nanomaterial surface functionalization ${ }^{[16]}$. Such a cloaking technique bestows nanoparticles with comprehensive properties and functions of the resident cells. A variety of cell membrane-coated nanoparticle systems have been developed with unique features 
and functions. Cell membrane from different cell types (for instance red blood cells, platelets, leukocytes, cancer cells, and bacteria) are incorporated on different synthetic nanoparticles (like polymeric nano-particles, gold nanoparticles, and silica nanoparticles) ${ }^{[17]}$. These biomimetic nanoparticles have been used in a wide range of biomedical applications including drug delivery, photodynamic therapy, detoxification, and vaccination ${ }^{[18]}$. Unlike cell membrane-coated nanoparticles, cell membrane cloaking materials exhibit the properties drastically different from those of spherical nanoparticles. More precisely, immune cell membrane cloaking technique has not been used on the modification of nano-materials. Whether electrospun nanofibers carrying immune cell membrane will protect MSCs residing in the nanofibers under pathologic circumstance of diabetic wound and improve their function is not clarified and needs to be investigated.

It has already been addressed that activated MSCs by interferon (IFN)- $\gamma$ exhibited stronger wound healing functions than MSCs themselves alone ${ }^{[19]}$. In addition, plenty of studies have demonstrated that the interactions between macrophages and MSCs supported biological function and behaviors of MSCs both in vitro and in vivo[20-22]. Considering that macrophages exert the immunomodulation on bone marrow MSCs (BMMSCs) through cell-cell contact ${ }^{[23]}$, whether macrophage cell membrane coated nanofiber scaffolds has in situ immunostimulation on BMMSCs with living material advantages is also worthy of exploration.

In this study, poly (lactic-co-glycolic acid) PLGA nanofiber, a scaffold material being approved for several biomedical applications including tissue engineering ${ }^{[24-26]}$, was electrospun with the cytomembrane from LPS/IFN- $\gamma$ activated macrophages and further used to load BMMSCs to construct a novel living material. We firstly addressed the biophysical properties of LPS/IFN- $\gamma$ activated RAW264.7 cell(a mouse M1 macrophage cell line model) membrane modified nanofibers (RCM-fibers) including the diameter, hydrophilicity and degradation rate. We further demonstrated that the RCM-fibers could improve the proliferation of BMMSCs and keratinocyte migration under oxidative stress in vitro. With the construction of RCMfiber-BMMSCs as a living material, it accelerated diabetic wound healing with better 
re-epithelialization, collagen remodeling, antioxidant stress and angiogenesis compared to the Un-fiber-BMMSCs. The effects of the RCM-fibers on gene expression of BMMSCs were detected by transcriptome analysis, which further validated that improved healing functions of RCM-fiber-BMMSCs were partially realized through CD200-CD200R (Scheme 1). Of note, we also demonstrated that the nanofibers modified by cytomembrane from LPS/IFN- $\gamma$ activated human THP-1 cells(a human M1 macrophage cell line model) (TCM-fibers) exhibited similar effects on promoting human BMMSC ( $\mathrm{hBMMSC}$ ) proliferation and keratinocyte migration upon oxidative stress. Being a living material, TCM-fiber-hBMMSCs also exhibited the advantages in diabetic wound healing. These results support that LPS/IFN- $\gamma$ activated macrophage cell membrane-modified nanofibers can in situ promote the biofunction of BMMSCs, making this novel living material promising in wound repair for human diabetes.

\section{Results}

\subsection{RCM-fibers promote the proliferation of BMMSCs and enhance their resistance} to oxidative stress.

Although extensive studies have demonstrated that BMMSCs were able to accelerate cutaneous wound healing[27], their vulnerability to environmental stress limits their long-term efficacy in clinic. We therefore prepared cell membrane from mouse macrophage cell line RAW264.7 treated with LPS and IFN- $\gamma$ in vitro and electrospun on PLGA nanofier scaffolds to prepare the RCM-fibers. The distribution of RAW264.7 cell membranes on the nanofibers was confirmed by coumarin (green) and Dil (red) staining, two lipophilic reporters for cell membrane (Fig. 1A). Western blotting analysis further demonstrated the enrichment of $\mathrm{Na}^{+} / \mathrm{K}^{+}$-ATPase (a plasma membrane-specific marker) and CD11c (a M1-like macrophage cell membrane specific marker) on the RCM-fibers whereas no $\mathrm{Na}^{+} / \mathrm{K}^{+}-\mathrm{ATPase}$ and $\mathrm{CD} 11 \mathrm{c}$ were detected on the Un-fibers (the nanofibers without cell membrane loading) (Fig. 1B). Comassie blue staining of protein profiles from the RCM-fibers was similar to those 
from cell membrane of LPS/IFN- $\gamma$ activated RAW264.7 (Fig. 1C). By using a scanning electron microscope (SEM), it was found that the RCM-fibers had a smooth outer surface and long fibrous morphology (Fig. 1D). The fibers loaded with cell membrane exhibited the diameters mainly in the range of 100-150 nm (Fig. 1E), which is aligned with the previous report ${ }^{[28]}$. In addition, the $\mathrm{RCM}$-fibers exhibited significantly improved hydrophilicity with lower water contact angel when compared to the Unfibers (Fig. 1F). The degradation rate of the Un-fibers was slower than the RCM-fibers (Fig. 1G)

Next we cultured BMMSCs with either the RCM-fibers or the Un-fibers exposed to $\mathrm{H}_{2} \mathrm{O}_{2}$ for 1,4 and 7 days, and determined the proliferation of BMMSCs through CFDA-SE staining for live cells. On day 1 , no obvious difference in green fluorescence was observed between two types of the nanofibers. However, on day 4 and day 7, more green fluorescence in the RCM-fibers was detectable when compared to that in the Un-fibers, demonstrating better proliferation of BMMSCs on the RCM-fibers (Fig. 1H). We also used the CCK-8 assay to determine the proliferation of BMMCs with either the RCM-fibers or the Un-fibers upon oxidative stress induced by $\mathrm{H}_{2} \mathrm{O}_{2}$. It was similar that the RCM-fibers supported the proliferation of BMMSCs with more extent when compared to the Un-fiber-BMMSCs group $(P<0.05)$ (Fig. 1I). Therefore, PLGA nanofibers electrospun with LPS/IFN- $\gamma$ treated RAW264.7 cell membrane (the RCM-fibers) is more favorable to support the proliferation of BMMCs than the Un-fibers especially under oxidative stress in vitro.

\subsection{RCM-fiber-BMMSCs accelerate wound closure accompanied by collagen remodeling and increased neovascularization in the wound areas of experimental diabetic mice.}

Since the RCM-fibers possessed more advantages in promote the proliferation of BMMSCs in vitro, we next seeds mouse BMMSCs on the RCM-fibers to prepare the RCM-fiber-BMMSCs as a living material and investigated their effects on wound repair in experimental diabetes mice (Fig. 2A). No difference in body weight and blood glucose levels was observed among mice receiving treatments (Fig. S1). It was 
apparent that there displayed the increase in wound closure rate in the RCM-fiberBMMSCs group than other four groups from day 5 (Fig. 2B). When calculating the remaining wound areas among different groups at day 15, it was found that the RCM-fiber-BMMSCs group showed the smallest wound area (2.94 $\pm 2.26 \%$ to Day 0$)$ compared to the Un-fiber-BMMSCs group $(13.09 \pm 1.90 \%)$, the RCM-fibers group $(24.05 \pm 2.81 \%)$, the Un-fibers group (37.28 $\pm 1.71 \%)$ and control group (38.58 $\pm 3.03 \%)$ with dramatic difference (Fig. 2C).

Wound re-epithelialization is one of the key features to evaluate the outcome of wound healing. Based on histological assessment of wound tissues after 15 days, it was showed that complete re-epithelialization occurred in the RCM-fiber-BMMSCs group when compared to the Un-fiber-BMMSCs and other groups with clear formation of epidermis, dermis and hypodermis layers similar to normal skins adjacent to the wounds (Fig. 2D). Epithelial formation can also be assessed by epithelial thickness. It was found that RCM-fiber-BMMSCs treatment led to complete epidermis formation within the early time period. The epidermal thickness was the most remote in the RCM-fiber-BMMSCs group $(0.02 \pm 0.006 \mathrm{~mm})$ among five groups (Fig. 2E) whereas other groups exhibited more epidermal thickness.

Masson's trichrome staining was further performed to visualize keratin and collagen composition in the wounds among different groups at day 15 . It was found that compared to control diabetic mice with only scattered, unstructured and a small amount of collagen deposition in the wound areas. By treatment with Un-fibers, RCM-fibers and Un-fiber-BMMSCs, there was increased collagen deposition with wound closure. However, RCM-fiber-BMMSCs group has less total collagen deposition than Un-fiber-BMMSCs group. (Fig. 3A). Picrosirius red staining has been performed to discriminate between mature and immature collagens in the wound areas. Immature collagen (regarded as Collagen Type 3) was labeled in green/yellow while organized and mature collagen fibers (regarded as Collagen Type 1) were labeled in orange/red. The RCM-fiber-BMMSCs treated wounds were largely composed of green/yellow collagen fibers (immature collagen deposition) and intermixed in a basket weave orientation, indicating normal healing. On the contrary, 
in the wounds from the Un-fibers group, the RCM-fibers group or the Un-fiberBMMSCs group, orange/red collagen fibers were dominant with parallel orientation implying the fibrosis of the local regions (Fig. 3B). When calculating the ratios of immature and mature collagen deposition among different groups, it was found that there was less mature Collagen 1 and more immature Collagen 3 in the wound regions treated with the RCM-fiber-BMMSCs living material (Fig. 3C).

It is recognized that BMMSCs alone are still vulnerable to oxidative stress during wound healing. Our in vitro assay demonstrated that the RCM-fiber-BMMSCs possessed more resistance to $\mathrm{H}_{2} \mathrm{O}_{2}$-induced oxidative stress with better proliferation capacity. To evaluate the efficacy of the RCM-fiber-BMMSCs in the resistance to oxidative stress in diabetic wound therapy, we stained the wound skins with 8-OHdG (a biomarker for DNA oxidative damage). It was found that the wounds treated with the RCM-fiber-BMMSCs had less 8-OHdG staining than either with the Un-fiberBMMSCs or other groups on day 7 post wounding (Fig. 3D, i). In addition, neovascularization in skin wounds is also one of key therapeutic objectives during wound healing. It was apparent that higher proportions of $\mathrm{CD} 31^{+}$cells, an indicator for angiogenesis in the wound areas were observed upon the treatment of the RCMfiber-BMMSCs than other groups at day 7 (Fig. 3D, iii), demonstrating the improved angiogenic ability of the RCM-fiber-BMMSCs. By using CK10 staining (differentiation marker keratin K10), we further validated better re-epithelialization with more CK10 expression in the wound areas from RCM-fiber-BMMSCs treated group when compared to other groups (Fig. 3D, ii). Collectively, these results support that the RCM-fiber-BMMSCs accelerate diabetic wound closure with the capability to collagen remodel and fasten the angiogenesis in the wound areas.

\subsection{RCM-fiber-BMMSCs treatment leads to decreased inflammation in diabetic wound regions.}

Since long-term and excessive inflammation prolongs the healing process of diabetic wounds, whether the RCM-fiber-BMMSCs also reduce the inflammatory responses during diabetic wound repair is further investigated. The percentages of 
$\mathrm{F} 4 / 80^{+} \mathrm{CD} 86^{+}$macrophages have been decreased in accordance with the lowest percentages of $F 4 / 80^{+}$TNF- $\alpha^{+}$cells in the wound areas upon RCM-fiber-BMMSCs treatment. On the contrary, the percentages of $\mathrm{F} 4 / 80^{+} \mathrm{CD} 206^{+}$macrophages were the highest on day 7 post-wounding when receiving RCM-fiber-BMMSCs treatment (Fig. 4A and 4B). We also determined the expression levels of IL-1B (a vital inflammatory cytokine) and IL-10 (an immunosuppressive cytokine) in wound regions by RT-PCR. It was found that $I L-16$ expression was prominently reduced in wound tissues on day 7 after RCM-fiber-BMMSCs treatment (Fig. 4C) whereas the level of IL-10 was exaggerated (Fig. 4D). These data demonstrate that RCM-fiberBMMSCs treatment is inclined to suppress local inflammation with more extent through the suppression of inflammatory cytokine production and induction of inhibitory cytokine (IL-10).

Being a promising immunotherapeutic living cell product, BMMSCs have been demonstrated to secrete extensive bioactive mediators among which tumor necrosis factor-induced protein 6 (TNFAIP6) ${ }^{[23,29]}$, Lipocalin-2 (LCn-2) ${ }^{[30-32]}$, chemokine (C-C motif) ligand $2(\mathrm{CCl} 2)^{[33-35]}$ and leukaemia inhibitory factor (Lif ${ }^{[36-38]}$ exert multiple functions including cell survival, immunomodulation, keratinocyte migration, angiogenesis and resistance to antioxidative stress respectively, which are critical for tissue repair in vivo. Therefore, expression levels of four genes were detected by RTqPCR in wound regions at the early time after the transplantation. It was significant that all four genes displayed remarkably increased expressing levels in the wounds when treated with the RCM-fiber-BMMSCs compared to the Un-fiber-BMMSCs whereas the wounds from the mice treated with the fibers without loading BMMSCs expressed low levels of four genes (Fig. 4E). These results further indicate that RCMfiber-loaded BMMSCs serves as a living material to induce less inflammatory environments in wound areas, which is largely associated with accelerating diabetic wound healing.

2.4 The RCM-fibers enhance the expression of wound healing related genes in BMMSCs facilitating kerotinocyte migration and less inflammation. 
To define the molecular signatures of BMMSCs regulated by the RCM-fibers, BMMSCs were cocultured with either the RCM-fibers or the Un-fibers in vitro and the transcriptomes were compared after the cocultures by using RNA-sequencing (Fig. 5A). Based on the RNA-seq results, there were 449 genes upregulated and 275 genes down-regulated in BMMSCs cocultured with the RCM-fibers when compared to those with the Un-fibers. GO enrichment scatter plot analysis revealed that the genes related to immunoregulation, cell proliferation, angiogenesis and cell migration were increased dramatically in RCM-fibers-cocultured BMMSCs (Fig. 5B). Consistent with the results from in vivo wound samples, four wound healing related genes we detected in wound areas including Ccl2, Lif, TNFAIP6 and Lcn2 were significantly higher in RCM-fiber-coclutured BMMSCs than those with Un-fibertreated BMMSCs (Fig. 5C), which was also validated by RT-qPCR (Fig. 5D).

Furthermore, in vitro assays were performed to verify the influence of the RCMfiber-BMMSCs on keratinocyte migration. Mouse keratinocyte cell line JB6 were cocultured with the RCM-fiber-BMMSCs or the Un-fiber-BMMSCs in the transwells with the addition of $\mathrm{H}_{2} \mathrm{O}_{2}$ to mimic oxidative stress (Fig. 5E). To avoid the interference of cell proliferation, JB6 cells were starved without the serum for $24 \mathrm{~h}$ before the injury. It was found that RCM-fiber-BMMSCs based migration of JB6 cells was faster than those with the Un-fiber-BMMSCs. Within $36 \mathrm{~h}$, the injury has nearly covered by JB6 cells with the incubation of the RCM-fiber-BMMSCs in the transwell whereas still open in Un-fiber-BMMSCs based group (Fig. 5F and 5G), which indicated that the RCM-fibers enhanced BMMSCs' ability to resist oxidative stress and promoted keratinocyte migration.

We also compared the effects of the RCM-fiber-BMMSCs and the Un-fiberBMMSCs on macrophage differentiation in vitro (Fig. 5H). RAW264.7 cells treated by LPS and IFN- $\gamma$ exhibited dramatic upregulation of CD86 and reduction of CD206 (named as RM1) (Fig. 5I, left). Incubation of RM1 cells with either the RCM-fiberBMMSCs or the Un-fiber-BMMSCs in the transwell dramatically reduced the expressions of CD86 and maintained CD206 expressions (Fig. 5I, middle and right). More significantly, incubation with the RCM-fiber-BMMSCs reduced the intracellular 
TNF- $\alpha$ level when compared to RM1 cells alones or those incubating with the Unfiber-BMMSCs (Fig. 5J). From in vitro investigations, we validate gene signatures of the BMMSCs regulated by the RCM-fibers most of which are associated with wound healing, supporting the roles of the RCM-fibers in BMMSCs to promote kerotinocyte migration and reduce the inflammation.

\subsection{CD200R-CD200 ligation is engaged in accelerated diabetic wound healing by the} RCM-fiber-BMMSCs.

From the RNA-seq, it was found that CD200 was dramatically upregulated on BMMSCs incubated with the RCM-fibers (Fig. 5C and 5D). To define whether CD200CD200R ligation is involved in the therapeutic effects of the RCM-fiber-BMMSCs on diabetic wound repair, we incubated the RCM-fiber-BMMSCs with anti-CD200R blocking antibody firstly and applied to the wound healing in diabetic mice. It was found that with the incubation of anti-CD200R antibody with the RCM-fiber-BMMSCs, the residual wound rates have been slowed down when compared to isotype control group (Fig. 6A). At day 15, the wound areas was $22.18 \pm 4.01 \%$ with anti-CD200R treatment, which was significantly higher than isotype control (3.98 $\pm 3.40 \%$ ) (Fig. 6C). H\&E staining of skin wounds on day 15 also demonstrated that there was more mature epidermis in RCM-fiber-BMMSCs group upon anti-CD200R treatment compared to that with isotype control group (Fig. 6B) with the increase in epidermis thickness (Fig. 6D). In addition, results from masson's trichrome staining also revealed more collagen deposition in anti-CD200R-RCM- fiber-BMMSCs treated tissues at day 15 when compared to IgG-RCM-fiber-BMMSCs treated wounds (Fig. $6 \mathrm{E})$.

In parallel, we have knocked down CD200 expressions in BMMSCs with shRNA and prepared the RCM-fiber-BMMSCs $(C D 200 K O)$ to evaluate the impacts on wound healing. Consistent with the results from anti-CD200R blocking assay on the RCMfiber-BMMSCs, the RCM-fiber-BMMSCs $(C D 200 K O)$ also slowed down wound closure when compared to the RCM-fiber-BMMSCs groups (Fig. 6F) with more residual wounds at day 5, day 10 and day 15 (Fig. 6H). There also existed more epidermis 
thickness in RCM-fiber-BMMSCs with CD200 deficiency compared to control BMMSCs (Fig. 6G and 6I) with more collagen deposition at day 15 (Fig. 6J). Picrosirius red staining showed that the wounds upon RCM-fiber-BMMSCs $(C D 200 K O)$ treatment had more Collagen 1 deposition and less Collagen 3 deposition when compared to the RCM-fiber-BMMSCs-control or untreated groups (Fig. 6K and 6L), which was similar to anti-CD200R treatment (Fig. S2). The expression levels of TNFAIP6 were lower in the wound regions upon RCM-fiber-BMMSCs (CD200KO) treatment than the counterpart group (Fig. 6M). Based on the results from anti-CD200R blocking assay and knockdown of CD200 in BMMSCs, we conclude that CD200R-CD200 ligation is largely engaged in accelerated diabetic wound healing mediated by the RCM-fibersBMMSCs.

\subsection{LPS/IFN $\gamma$ activated THP-1 cell membrane-modified nanofiber scaffolds improve} the effects of human BMMSCs in diabetic skin wound healing.

Our aforementioned results indicated that mouse-derived RCM-fibers-BMMSCs were apparently able to ameliorate wound healing process. We further modified the nanofibers with cell membrane from human derived THP-1 cells pre-treated with LPS and IFN $\gamma$ which exhibited similar properties to the RCM-fibers (Fig. 7A-D). Electrospun nanfibers loading human BMMSCs (hBMMSCs) were prepared similarly as a living material named as the TCM-fiber-hBMMSCs. Their effects on diabetic wound healing were evaluated as well. It was evident that the TCM-fiber-hBMMSCs also accelerated wound closure with the smallest residual wound area especially at day 15 when compared to other groups (Fig. 8A and 8B). Results from H\&E staining indicated that the differentiation of epidermal cells and the formation of hair follicles were enhanced upon the treatment of the TCM-fiber-hBMMSCs (Fig. 8C and 8D). In wound regions there showed faster collagen remodeling (Fig. 8E), increased Collagen 3 deposition and less Collagen 1 deposition in TCM-fiber-hBMMSCs treated group (Fig. $8 \mathrm{~F}$ and $8 \mathrm{G}$ ). Therefore, similar to the RCM-fiber-BMMSCs, the TCM-fiberhBMMSCs living material also promotes wound healing with less epidermis thickness and more collagen deposition close to normal skins. 


\section{Discussion}

In the present study, we have modified nanofibers with LPS/IFN $\gamma$ activated macrophage cell membrane which is demonstrated to promote the proliferation of BMMSCs and enhance their resistance to oxidative stress. BMMSCs loading on cell membrane-nanofibers also accelerated wound closure in diabetes mice. LPS/IFN $\gamma$ treated macrophage cell membrane-modified nanofibers loading BMMSCs thus become an alternative potential immuno-activated living material to improve the clinic applications of BMMSCs in the future.

Biomaterials have been widely used in tissue regeneration. An ideal biomaterial possesses essential properties such as hierarchical architecture and extracellular matrices (ECM) similar to native tissues ${ }^{[39]}$. Nanofibers have been widely used in regenerative engineering due to their feasibility in modification, making them more interactive with tissue environment and providing regenerative signals to the tissues ${ }^{[40]}$. Among multiple modifications, cell membrane modified nanofibers exhibit more advantages due to strong hydrophilicity, high velocity of degradation and fruitfulness of bona fide surface molecules anchoring on live cells ${ }^{[28]}$. In our study, we used LPS/IFN $\gamma$ activated macrophage cell lines-derived cytomembrane to modify nanofibers. Both mouse RAW264.7 and human THP-1 cell lines belong to monocyte lineages that can be easily activated to different macrophage subtypes based on conditional cytokines such as LPS/IFN $\gamma$ for M1-like macrophages and IL-4 for M2-like macrophages ${ }^{[41]}$. Herein the reason we used LPS/IFN $\gamma$ for stimulation of cell lines was largely because it has been reported as the optimized condition for the proliferation and functions of BMMSCs. In fact macrophage-MSC crosstalk in part generates paracrine signals to affect cell differentiation which is related to the outcome of tissue regeneration. Although exosomes from MSCs can affect the differentiation of inflammatory (M1) and regenerative (M2) macrophages ${ }^{[42]}, \mathrm{M} 1$ or $\mathrm{M} 2$ macrophages can also secrete multiplex bioactive molecules to guide MSC differentiation and affect their survival and proliferation ${ }^{[43]}$. We have used LPS/IFN $\gamma$ activated macrophage cell membrane to modify the nanfibers because previous studies have 
already demonstrated the efficacy of M1 macrophages to support the survival and proliferation of BMMSCs. In our study, TNFAIP6 could be induced in BMMSCs when they were loaded on LPS/IFN $\gamma$ activated macrophage (M1) cell membrane-containing nanofibers whereas little was detectable in BMMSCs with nanofibers only or with IL4 activated macrophage (M2) cell membrane-containing nanofibers (Fig. S3). This can further support the roles of M1-like macrophage in modulating regenerative properties of BMMSCs.

What is more, based on our results the nanofibers modified with LPS/IFN $\gamma$ activated macrophage cell membrane exaggerate the resistance of BMMSCs to oxidative stress. When BMMSCs are transplanted to the wound regions, hypoxic environments are usually generated with increased reactive oxygen species $(\mathrm{ROS})^{[44]}$ which may inhibit the proliferation and immunoregulation of $\mathrm{BMMSCS}^{[45]}$ whereas increase senescence and adipogenic differentiation of $\mathrm{BMMSCs}^{[46]}$. In our study, the RCM-fibers not only favor BMMSC proliferation in oxidative stress (Fig. $1 \mathrm{H}$ and $1 \mathrm{I}$ ), but also promote the migration of kerotinocytes in vitro (Fig. 5F and 5G). This was consistent with rapid re-epithelialization and wound closure in diabetic mice. RCM-modified nanofibers thus largely augment the resistance of BMMSC to harmful microenvironments.

When the RCM-fibers were used to load BMMSCs (RCM-fiber-BMMSCs) for in vivo diabetic wound healing in mice, more advantages were shown up including epidermal formation, collagen remodeling and angiogenesis. These events are all key steps for skin regeneration that are interacted. Firstly, keratinocytes activation and differentiation are strongly associated with epidermal formation ${ }^{[47]}$. In our study we have observed incomplete differentiation of keratinocytes in the Un-fibers, RCMfibers and Un-fiber-BMMSCs treated wounds. Keratinocyte migration is also accelerated in RCM-fiber-BMMSCs treated wounds leading to complete remodeling within the speculated time period. Secondly, the RCM-fiber-BMMSCs also promoted rapid accumulation of epidermal cells in the wound areas developing mature epithelial structures such as hair follicles, making the skin regenerated more rapidly. Thirdly, during initial wound healing there was increased fibroblast proliferation 
followed by the deposition of immature collagen 3, which is mainly applied for wound closure and avoid further damage ${ }^{[48,49]}$. Therefore collagen remodeling and collagen composition in wound areas are also key features to evaluate the regeneration of the wounds. In this study, we have observed less total collagen and chiefly immature collagen deposition in the wounds treated with the RCM-fiberBMMSCs. Fourthly, since defective angiogenesis is also present in diabetic wounds due to enhanced mitochondrial ROS production ${ }^{[46]}$, we have also observed more angiogenesis in wound areas upon RCM-fiber-BMMSCs treatment. All these data strong support that the RCM-fibers remodel BMMSCs with more features to promote diabetic wound regeneration. The RCM-fiber-BMMSCs thus display the advantages in maintaining the cell functionality and remodeling wound environment, making this living material more promising in wound repair.

To further define the molecular signatures of BMMSCs induced by the RCMfibers, we have performed the RNA-seq to compare the gene expression profiles of BMMSCs upon the RCM-fibers and the Un-fibers treatments. It was found that the expressions of skin regeneration-related genes including CCI2, Lif, TNFAIP6 and Lcn2 were dramatically increased both in vitro and in vivo. TNFAIP6 improves immune regulation and accelerates collagen remodeling ${ }^{[23,29]}$. Lcn2 promotes BMMSCs proliferation in oxidative stress and keratinocytes migration ${ }^{[30-32]}$. Lif protects against diabetic wound from oxidative stress ${ }^{[36-38]}$. $\mathrm{Ccl} 2$ accelerates vascularization in the wounds ${ }^{[33-35]}$. The increase in the expressions of four genes in RCM-fiber-loaded BMMSCs is extremely consistent with high efficacy of wound closure and related skin regeneration in diabetic mice. The in situ immune-stimulation on BMMSCs by the cell membrane both in vitro and in vivo largely favor the bio-functionality of this living material.

Moreover, from RNA-seq analysis we have identified the upregulation of CD200 in BMMSCs that was strongly associated with RCM-fiber-BMMSCs mediated wound healing. Both anti-CD200R treatment and silencing CD200 in BMMSCs restrained the wound healing effects when compared to RCM-fiber-BMMSCs treatments. Recent research revealed that direct contact with M1-type macrophages not only 
increased production of TNFAIP6 but also upregulated CD200 expression on $\mathrm{BMMSCs}^{[23]}$. CD200 is widely expressed in DC, thymocytes, B cells, MSCs, the central nervous system nerves, and other hematopoietic and non-hematopoietic tissuederived cells ${ }^{[50]}$. It can interact with its ligand CD200R to trigger the recruitment and phosphorylation of Dok-2 and 1, which then bind to RasGAP [51]. In macrophages and mast cells, this cascade has been shown to inhibit the phosphorylation of ERK, P38, and JNK, and the activation of myeloid cells ${ }^{[52]}$. Although we did not determine the cell types expressing CD200R in wound regions, the regulatory roles of CD200CD200R signaling defined in other cell types are consistent with the observation that the RCM-fiber-BMMSC treatment led to low inflammation in the wound areas. The exact mechanisms will be investigated further.

Last but not the least, we have also demonstrated the efficacy of humanderived BMMSCs in promoting diabetic wound healing in mice when loaded on electrospun nanofibers with LPS/IFN- $\gamma$ activated M1-like THP-1 macrophage cytomembrane. hBMMSCs can be either injected topically or applied with various matrix including nanofiber scaffolds, hydrogels, extracellular matrix derivatives and dermal substitutes ${ }^{[53,54]}$. They are demonstrated to promote skin wound healing by multiple mechanisms including releasing bioactive molecules, reducing the inflammation, stimulating resident cells and remodeling extracellular matrix ${ }^{[55]}$. Herein using the TCM-fibers, hBMMSCs have accelerated the repairment of diabetic wounds, which is similar to mice-derived RCM-fiber-BMMSCs. We have also observed the improvement of the TCM-fibers on hBMMSCs proliferation (Fig. 7). The TCM-fiber-BMMSCs have promoted human keratinocyte migration under oxidative stress (Fig. 7G and 7H). Cell membranes derived from both human and mice LPS/IFN$\gamma$ activated M1-like macrophages modulate BMMSCs with similar patterns. Similar therapeutic effects of RCM-fiber-BMMSCs and TCM-fiber-hBMMSCs living materials on diabetic wound healing to some extent validate the universality of M1-like macrophage membrane modified nanofibers as an alternative scaffolds for BMMSCs in clinical application of wound repair in the future. 


\section{Conclusion}

Collectively, we have prepared the nanofibers electrospun with LPS/IFN- $\gamma$ activated macrophage cell membrane to immunostimulate human or mice BMMSCs as a novel living material for diabetic skin regeneration. Our results reveal that cell membrane coated electrospun nanofibers not only augment the proliferation of BMMSCs under oxidative stress in vitro, but also make BMMSCs accelerate wound healing in diabetic mice through favoring immunoregulation, keratinocyte migration, collagen remodeling, antioxidant stress and neovascularization. This is in part due to upregulation of CD200 on BMMSCs induced by cell membrane-loaded nanofibers, leading to CD200R-CD200 ligation and subsequently gene expressions change. We have thus provided an alternative engineering way of nanofiber scaffolds to develop a novel living material for the improvement in diabetic chronic wound management.

\section{Materials and methods}

\subsection{Cell culture}

Human bone marrow-derived mesenchymal stem cells (hBMMSCs) and mouse BMMSCs were purchased from Cyagen Biosciences Co., Ltd (Guangzhou, China). RAW264.7, JB6 cell, THP-1 cell and HACAT cell were purchased from Beina Biotechnology Co., Ltd (Guangzhou, China). All cells were maintained according to the company's instructions. RAW264.7 cells were treated with $1 \mu \mathrm{g} / \mathrm{mL}$ Lipopolysaccharides (LPS) (Sigma-Aldrich) and $20 \mathrm{ng} / \mathrm{mL}$ Interferon (IFN)- $\gamma$ (SigmaAldrich) for $12 \mathrm{~h}$. THP-1 cell were treated with $1000 \mathrm{ng} / \mathrm{mL}$ LPS (Sigma-Aldrich) and $20 \mathrm{ng} / \mathrm{mL}$ hIFN- $\gamma$ (Sigma-Aldrich) for $24 \mathrm{~h}$ after pre-treatment with $200 \mathrm{ng} / \mathrm{mL}$ Phorbol-12-myristate-13-acetate (PMA) (Sigma-Aldrich) for $6 \mathrm{~h}$.

\subsection{Preparation and identification of cell membrane-modified PLGA nanofibers}

PLGA balls (85:15; Polylactic acid (PLA: PGA) were dissolved in organic solvents of dimethylformamide and tetrahydrofuran. The block PLGA copolymer solution was loaded into a $5 \mathrm{ml}$ syringe. An electric field is generated between the anode and the rectangular stainless steel plate with an $18 \mathrm{kV}$ high voltage power supply. The PLGA copolymer solution is ejected from the syringe under the accurate infusion control 
pump and sprayed onto the stainless steel plate with tin foil paper through the electrostatic field ${ }^{[56]}$.

LPS/IFN- $\gamma$-treated and untreated RAW264.7 or THP-1 cells were ultrasonicated and cell membrane was collected after the treatment. Cell membrane was harvested as previously reported. Briefly, cells were grown in T-175 culture flasks to full confluency and detached by scrapping the culture flask surfaces. Cells were suspended in a hypotonic lysing buffer containing $20 \mathrm{mM}$ Tris- $\mathrm{HCl}(\mathrm{pH}=7.5), 10 \mathrm{mM}$ $\mathrm{KCl}, 2 \mathrm{mM} \mathrm{MgCl}$, and EDTA-free mini protease inhibitor tablet (Solarbio), and disrupted using a dounce homogenizer with a tight-fitting pestle. The cell suspension was subjected to 20 passes and centrifuged at $20000 \mathrm{~g}$ for $20 \mathrm{~min}$. The supernatant was collected and centrifuged again at $100000 \mathrm{~g}$ for $45 \mathrm{~min}$. The pellet was collected and used as purified cell membrane for subsequent experiments. Cell membrane suspension was added to the nanofibers and soaked for 30 minutes. The solvent was discarded and the nanofiber scaffolds were rinsed with PBS. The morphology of RCM-fibers (with LPS/IFN- $\gamma$-treated RAW264.7 cell membrane) and TCM-fibers (with LPS/IFN- $\gamma$-treated THP-1 cell membrane) was analyzed by a scanning electron microscopy (SEM) (Carl Zeiss). The diameter and size distribution of the nanofibers were calculated by measuring at least 100 fibers which were selected randomly from five independent samples.

To prepare PLGA copolymer solution, $10 \mu \mathrm{L}$ coumarin fluorescent dyes $(1 \mu \mathrm{M})$ (Sigma-Aldrich) was added to PLGA copolymer solution according to the manufacturer's instructions. $1 \mathrm{~mL}$ 1,1'-dioctadecyl-3,3,3',3'tetramethylindocarbocyanine perchlorate (DiL) (5 $\mu \mathrm{M}$, Sigma-Aldrich) was added on $1 \mathrm{~cm}^{2} \mathrm{RCM}$ fibers and Un-fibers according to the product description. The water contact angles of modified nanofibrous membranes were measured using water contact angle analyzer (FTA200). The samples were put on the testing plate and a drop of distilled water was carefully placed on the surface of the samples. The measurements were carried out five times for each sample. 


\subsection{Western blotting}

The RCM-fibers were decomposed by ultrasound for 5 min with the addition of protease inhibitor. The proteins were collected and subjected to sodium dodecyl sulfate-polyacrylamide gel electrophoresis (SDS-PAGE). Proteins were transferred to PVDF membrane (ThermoFisher Scientific). After blocking with phosphate buffer saline (PBS) containing $5 \%$ bovine plasma albumin (BSA), the membranes were incubated with anti-mouse CD11c (1:500) (Abcam) and anti-mouse $\mathrm{Na}^{+} / \mathrm{K}^{+}$ATPase (1:300) (Abcam) primary antibodies overnight at $4{ }^{\circ} \mathrm{C}$. The membranes were further incubated with Horseradish Peroxidase (HRP)-conjugated secondary antibody (1:2000) (Abcam). After washing four times with TBS-T, immunopositive bands were visualized by chemiluminescence detection system (Merck Millipore).

\subsection{Flow cytometry}

The cells were washed and incubated with fluorescence conjugated antibodies including PE-anti-mouse CD86 (Abcam) and FITC-anti-mouse CD206 (Abcam) for surface staining, and FITC-anti-mouse TNF- $\alpha$ antibody (Abcam) for intracellular staining according to the manufacturer's instructions (BD Biosciences). Cells were washed, resuspended in PBS and acquired on BD FACSverse flow cytometer (BD Biosciences). The data were analyzed by Flowjo software (version 7.6.1) (Tree Star Inc.).

\subsection{Quantitative real-time PCR}

Cells were mixed with TRIzol Reagent (Invitrogen). Total RNA was extracted and reversely transcribed into cDNA by using QuantiTect Reverse Transcription Kit (Qiagen) in accordance with the manufacturer's instructions. Polymerase chain reaction (PCR) was performed by using SYBR Green qPCR Master Mix (MedChemExpress). Gene expression levels in individual samples were calculated based on the $2^{-\triangle \triangle C T}$ according to the threshold cycle (CT) values of target genes and the house-keeping gene. The primers for target genes and GAPDH as the house- 
keeping gene were listed in Table S1.

\subsection{Cell migration assay}

Cell migration assay was performed by using six-well transwell plate (Corning Inc.). After $24 \mathrm{~h}$ of cell attachment, JB6 cells were serum-starved overnight. To mimic oxidative stress, JB6 cells were exposed to $200 \mu \mathrm{M} \mathrm{H} \mathrm{H}_{2} \mathrm{O}_{2}$ for $2 \mathrm{~h}$ and were furhter incubated with the RCM-fiber-BMMSCs. Wound closure was imaged at $12 \mathrm{~h}, 24 \mathrm{~h}$ and $36 \mathrm{~h}$, respectively. The wound area was quantified using ImageJ soft-ware to calculate the percentages of wound closure.

\subsection{BMMSCs proliferation}

$2 \times 10^{4}$ third-generation BMMSCs or hBMMSCs were seeded and exposed to 600 $\mu \mathrm{M} \mathrm{H}_{2} \mathrm{O}_{2}$ for 2-6 h. BMMSCs were incubated with the RCM-fibers or the Un-fibers for 1, 4, and 7 days and stained with CFDA-SE (ThermoFisher Scientific). CCK8 assay was also applied to investigate the proliferation of BMMSCs on the RCM-fibers and the Un-fibers. After 1, 4 or 7 days, the supernatant was discarded and $100 \mu \mathrm{L}$ of CCK-8 solution was added to each well, and incubated for another $4 \mathrm{~h}$. The OD values of each well were measured by an ELISA assay reader (Infinite M200) at $450 \mathrm{~nm}$ wave length.

\subsection{Knockdown of CD200 in BMMSCs}

BMMSCs were transfected with CD200-specific or nonspecific control short hairpin RNA (shRNA) (Ribobio Biotechnology) by using transfection reagent (Ribobio Biotechnology) according to the manufacturer's protocol. The knockdown efficiency of CD200 was detected by RT-qPCR after $48 \mathrm{~h}$.

\subsection{Surgical procedure}

6-week-old C57BL/6J male mice were purchased from Tengxin Technology Co. Ltd and maintained under a specific pathogen-free (SPF) mouse facility. The mice were continuously fed on high-fat and high sugar diet for 4 weeks. On day 19, 
C57BL/6J mice were intraperitoneally injected with $100 \mu \mathrm{L}$ streptozotocin (STZ) (50 $\mathrm{mg} / \mathrm{kg}$, Sigma-Aldrich) for 3 days. On day 28, blood glucose levels were surveyed in the blood using a glucometer (Sinocare, China). Diabetic Mice were generated when blood glucose levels consistently exceeded $14 \mathrm{mmol} / \mathrm{L}$ (Fig. S1A and S1B).

Biopsy punches $(1.0 \mathrm{~cm}$ diameter) were generated by removing epidermis and dermis. The Un-fiber, RCM-fiber, Un-fiber-BMMSCs and RCM-fiber-BMMSCs with similar diameter were applied on the wound beds while mice in the control group had no fiber treatment. Wound closure was determined through picture collection at Days 0,5 and 15 respectively, and analyzed by ImageJ software. In some experiments, anti-mouse CD200R (20 ng/mL) (Abcam) or IgG antibodies were mixed with RCM-fiber-BMMSCs before subsequent experiment. In addition, BMMSCs with CD200 deficiency were used in some experiments. The experiments were approved by the Institute Animal Ethics Committee of Shanghai Jiaotong University School of Medicine and the Ethics Committee of Zunyi Medical Affiliated University Hospital.

\subsection{Histological analysis and immunostaining}

Mice were sacrificed after 15 days. The wound tissue was fixed in $10 \%$ formalin and embedded in paraffin. The sections of diabetic wound were stained with hematoxylin and eosin (H \& E), Masson's trichrome and picrosirius red. Images were scanned by ServiceBio Company. Picrosirius red pictures were photographed by polarized light microscope in Servicebio Company. The collagen content was quantified using ImageJ v1.8.0.

For immunostaining, wound sections were dewaxed and rinsed with PBS. For antigen retrieval, tissue sections were treated with sodium citrate buffer at $95^{\circ} \mathrm{C}$ for $10 \mathrm{~min}$, followed by permeabilization with $0.1 \%$ Triton $\mathrm{X}-100$ for $10 \mathrm{~min}$. After blocking in 2\% BSA in PBST (PBS + 0.1\% Tween 20), tissue sections were incubated with 8-OHdG (Abcam; 1:200), anti-mouse CD31 (Abcam; 1:300), anti-CK10 (Abcam; 1:100), anti-mouse F4/80 (Abcam; 1:100), anti-mouse CD86 (Abcam; 1:100) or antimouse CD206 (Abcam; 1:100) antibodies for $8 \mathrm{~h}$ at $4{ }^{\circ} \mathrm{C}$. Goat anti-mouse FITC secondary antibody (Abcam;1:200) and goat anti-rabbit TRITC secondary antibody 
(Abcam; 1:400) were added further for $2 \mathrm{~h}$ at room temperature. The fluorescence pictures were collected through a confocal fluorescence microscope (Leica SPII) with 20x magnification.

\subsection{Statistical analysis}

Data were presented as mean \pm standard error of mean (SEM) or standard deviation (SD). Graphpad Prism 7.0 was used to analyze the differences between the experimental groups using Student t-test, one way and two-way ANOVA tests followed by a Tukey post hoc test for pairwise comparison. $P<0.05$ was considered significant.

\section{Acknowledgements}

We acknowledge the support of Shanghai Institute of Immunology, Shanghai Key Laboratory for Prevention and Treatment of Bone and Joint Diseases, Shanghai Institute of Traumatology and Orthopaedics and Special Key Laboratory of Gene Detection and Therapy \& Base for Talents in Biotherapy of Guizhou Province.

\section{Competing interests}

All authors declare that the research was conducted in the absence of any commercial or financial relationships that could be construed as a potential conflict of interest.

\section{Author Contributions}

Ying Wang and Shaoying Gao conceived and designed the experiments. Shaoying Gao and Tao Chen performed the experiments. Zhen Wang and Wenguo Cui performed and supervised the experiments of biomaterial preparation. Ping Ji provided the technical support. Lin Xu supervised the experiment. Ying Wang and Shaoying Gao analysed the data and wrote the manuscript. All authors read and approved the final manuscript. 


\section{Availability of data and materials}

All data are available in the main text or the additional materials.

\section{Funding}

This work was supported by Key Project from National Natural Science Foundation of China (81930051), Science and Technology Fund Project of Guizhou Provincial Health Commission (gzwjkj2020-1-116), Program for High level innovative talents in Guizhou Province (QKH-RC-2016-4031).

\section{References}

[1] Service RF. Synthetic biology. Synthetic biologists design 'living materials' that build themselves. Science. 2014. 343(6178): 1421.

[2] Rodrigo-Navarro A, Sankaran S, Dalby MJ, Campo AD, Salmeron-Sanchez M. Engineered living biomaterials. Nature Reviews Materials. 2021.

[3] He F, Ou Y, Liu J, et al. 3D Printed Biocatalytic Living Materials with DualNetwork Reinforced Bioinks. Small. 2022. 18(6): e2104820.

[4] Xin A, Su Y, Feng S, et al. Growing Living Composites with Ordered Microstructures and Exceptional Mechanical Properties. Adv Mater. 2021. 33(13): e2006946.

[5] Winnacker M. Recent advances in the synthesis of functional materials by engineered and recombinant living cells. Soft Matter. 2017. 13(38): 66726677.

[6] Gartner Z, Hughes A. Getting the measure of living biomaterials. Nature. 2019. 572(7767): 38-39.

[7] Sedel L, Petite H, Bizot P, Nizard R, Meunier A. [Biomaterials and the living system]. Bull Acad Natl Med. 1999. 183(3): 541-51; discussion 552-4.

[8] Gurtner GC, Werner S, Barrandon Y, Longaker MT. Wound repair and regeneration. Nature. 2008. 453(7193): 314-21.

[9] Hunt TK, Burke J, Barbul A, Gimbel ML. Wound healing. Science. 1999. 284(5421): 1775. 
[10] Boulton AJ, Vileikyte L, Ragnarson-Tennvall G, Apelqvist J. The global burden of diabetic foot disease. Lancet. 2005. 366(9498): 1719-24.

[11] Shin YC, Lee JH, Jin L, et al. Stimulated myoblast differentiation on graphene oxide-impregnated PLGA-collagen hybrid fibre matrices. J Nanobiotechnology. 2015. 13: 21.

[12] Vogel V, Sheetz M. Local force and geometry sensing regulate cell functions. Nat Rev Mol Cell Biol. 2006. 7(4): 265-75.

[13] Zhang Z, Gupte MJ, Jin X, Ma PX. Injectable Peptide Decorated Functional Nanofibrous Hollow Microspheres to Direct Stem Cell Differentiation and Tissue Regeneration. Adv Funct Mater. 2015. 25(3): 350-360.

[14] Tunuguntla RH, Bangar MA, Kim K, et al. Bioelectronic light-gated transistors with biologically tunable performance. Adv Mater. 2015. 27(5): 831-6.

[15] Williams DF. On the nature of biomaterials. Biomaterials. 2009. 30(30): 5897909.

[16] Gao W, Fang RH, Thamphiwatana S, et al. Modulating antibacterial immunity via bacterial membrane-coated nanoparticles. Nano Lett. 2015. 15(2): 1403-9.

[17] Hu CM, Fang RH, Wang KC, et al. Nanoparticle biointerfacing by platelet membrane cloaking. Nature. 2015. 526(7571): 118-21.

[18] Ding H, Lv Y, Ni D, et al. Erythrocyte membrane-coated NIR-triggered biomimetic nanovectors with programmed delivery for photodynamic therapy of cancer. Nanoscale. 2015. 7(21): 9806-15.

[19] García JR, Quirós M, Han WM, et al. IFN- $\gamma$-tethered hydrogels enhance mesenchymal stem cell-based immunomodulation and promote tissue repair. Biomaterials. 2019. 220: 119403.

[20] Zhang Y, Böse T, Unger RE, Jansen JA, Kirkpatrick CJ, van den Beucken J. Macrophage type modulates osteogenic differentiation of adipose tissue MSCs. Cell Tissue Res. 2017. 369(2): 273-286.

[21] Zhang Q, Hwang JW, Oh JH, et al. Effects of the fibrous topography-mediated macrophage phenotype transition on the recruitment of mesenchymal stem cells: An in vivo study. Biomaterials. 2017. 149: 77-87. 
[22] Yu B, Sondag GR, Malcuit C, Kim MH, Safadi FF. Macrophage-Associated Osteoactivin/GPNMB Mediates Mesenchymal Stem Cell Survival, Proliferation, and Migration Via a CD44-Dependent Mechanism. J Cell Biochem. 2016. 117(7): 1511-21.

[23] Li Y, Zhang D, Xu L, et al. Cell-cell contact with proinflammatory macrophages enhances the immunotherapeutic effect of mesenchymal stem cells in two abortion models. Cell Mol Immunol. 2019. 16(12): 908-920.

[24] Agrawal CM, Ray RB. Biodegradable polymeric scaffolds for musculoskeletal tissue engineering. J Biomed Mater Res. 2001. 55(2): 141-50.

[25] Behravesh E, Yasko AW, Engel PS, Mikos AG. Synthetic biodegradable polymers for orthopaedic applications. Clin Orthop Relat Res. 1999. (367 Suppl): S118-29.

[26] Athanasiou KA, Niederauer GG, Agrawal CM. Sterilization, toxicity, biocompatibility and clinical applications of polylactic acid/polyglycolic acid copolymers. Biomaterials. 1996. 17(2): 93-102.

[27] He X, Dong Z, Cao Y, et al. MSC-Derived Exosome Promotes M2 Polarization and Enhances Cutaneous Wound Healing. Stem Cells Int. 2019. 2019: 7132708.

[28] Chen W, Zhang Q, Luk BT, et al. Coating nanofiber scaffolds with beta cell membrane to promote cell proliferation and function. Nanoscale. 2016. 8(19): 10364-70.

[29] Qi Y, Jiang D, Sindrilaru A, et al. TSG-6 released from intradermally injected mesenchymal stem cells accelerates wound healing and reduces tissue fibrosis in murine full-thickness skin wounds. J Invest Dermatol. 2014. 134(2): 526-537.

[30] Halabian R, Tehrani HA, Jahanian-Najafabadi A, Habibi Roudkenar M. Lipocalin-2-mediated upregulation of various antioxidants and growth factors protects bone marrow-derived mesenchymal stem cells against unfavorable microenvironments. Cell Stress Chaperones. 2013. 18(6): 785-800.

[31] Bahmani B, Roudkenar MH, Halabian R, Jahanian-Najafabadi A, Amiri F, Jalili 
MA. Lipocalin 2 decreases senescence of bone marrow-derived mesenchymal stem cells under sub-lethal doses of oxidative stress. Cell Stress Chaperones. 2014. 19(5): 685-93.

[32] Miao Q, Ku AT, Nishino Y, et al. Tcf3 promotes cell migration and wound repair through regulation of lipocalin 2. Nat Commun. 2014. 5: 4088.

[33] Whelan DS, Caplice NM, Clover A. Mesenchymal stromal cell derived CCL2 is required for accelerated wound healing. Sci Rep. 2020. 10(1): 2642.

[34] Khan B, Rangasamy S, McGuire PG, Howdieshell TR. The role of monocyte subsets in myocutaneous revascularization. J Surg Res. 2013. 183(2): 963-75.

[35] Stamatovic SM, Keep RF, Mostarica-Stojkovic M, Andjelkovic AV. CCL2 regulates angiogenesis via activation of Ets-1 transcription factor. J Immunol. 2006. 177(4): 2651-61.

[36] Dong S, Zhen F, Xu H, Li Q, Wang J. Leukemia inhibitory factor protects photoreceptor cone cells against oxidative damage through activating JAK/STAT3 signaling. Ann Transl Med. 2021. 9(2): 152.

[37] Negoro S, Kunisada K, Fujio Y, et al. Activation of signal transducer and activator of transcription 3 protects cardiomyocytes from hypoxia/reoxygenation-induced oxidative stress through the upregulation of manganese superoxide dismutase. Circulation. 2001. 104(9): 979-81.

[38] Xu J, Li Z, Xu P, Yang Z. Protective effects of leukemia inhibitory factor against oxidative stress during high glucose-induced apoptosis in podocytes. Cell Stress Chaperones. 2012. 17(4): 485-93.

[39] Hu C, Ahmad T, Haider MS, et al. A thermogelling organic-inorganic hybrid hydrogel with excellent printability, shape fidelity and cytocompatibility for 3D bioprinting. Biofabrication. 2022. 14(2).

[40] Yuan Z, Sheng D, Jiang L, et al. Vascular Endothelial Growth Factor-Capturing Aligned Electrospun Polycaprolactone/Gelatin Nanofibers Promote Patellar Ligament Regeneration. Acta Biomater. 2022. 140: 233-246.

[41] Chanput W, Mes JJ, Wichers HJ. THP-1 cell line: an in vitro cell model for immune modulation approach. Int Immunopharmacol. 2014. 23(1): 37-45. 
[42] Wang R, Ji Q, Meng C, et al. Role of gingival mesenchymal stem cell exosomes in macrophage polarization under inflammatory conditions. Int Immunopharmacol. 2020. 81: 106030.

[43] He XT, Li X, Yin Y, Wu RX, Xu XY, Chen FM. The effects of conditioned media generated by polarized macrophages on the cellular behaviours of bone marrow mesenchymal stem cells. J Cell Mol Med. 2018. 22(2): 1302-1315.

[44] Wang H, Jiang H, Van De Gucht M, De Ridder M. Hypoxic Radioresistance: Can ROS Be the Key to Overcome It. Cancers (Basel). 2019. 11(1).

[45] Hou J, Han ZP, Jing YY, et al. Autophagy prevents irradiation injury and maintains stemness through decreasing ROS generation in mesenchymal stem cells. Cell Death Dis. 2013. 4(10): e844.

[46] Deng Z, Wang W, Xu X, et al. Biofunction of Polydopamine Coating in Stem Cell Culture. ACS Appl Mater Interfaces. 2021. 13(9): 10748-10759.

[47] van den Bogaard EH, Podolsky MA, Smits JP, et al. Genetic and pharmacological analysis identifies a physiological role for the AHR in epidermal differentiation. J Invest Dermatol. 2015. 135(5): 1320-1328.

[48] Priya SG, Jungvid H, Kumar A. Skin tissue engineering for tissue repair and regeneration. Tissue Eng Part B Rev. 2008. 14(1): 105-18.

[49] Schreurs $M$, Suttorp CM, Mutsaers $\mathrm{H}$, et al. Tissue engineering strategies combining molecular targets against inflammation and fibrosis, and umbilical cord blood stem cells to improve hampered muscle and skin regeneration following cleft repair. Med Res Rev. 2020. 40(1): 9-26.

[50] Zhao Y, Su G, Wang Q, Wang R, Zhang M. The CD200/CD200R mechanism in mesenchymal stem cells' regulation of dendritic cells. Am J Transl Res. 2021. 13(8): 9607-9613.

[51] Liu JQ, Hu A, Zhu J, Yu J, Talebian F, Bai XF. CD200-CD200R Pathway in the Regulation of Tumor Immune Microenvironment and Immunotherapy. Adv Exp Med Biol. 2020. 1223: 155-165.

[52] Zhang S, Cherwinski H, Sedgwick JD, Phillips JH. Molecular mechanisms of CD200 inhibition of mast cell activation. J Immunol. 2004. 173(11): 6786-93. 
[53] Mahmoudian-Sani MR, Rafeei F, Amini R, Saidijam M. The effect of mesenchymal stem cells combined with platelet-rich plasma on skin wound healing. J Cosmet Dermatol. 2018. 17(5): 650-659.

[54] Sánchez-Sánchez R, Brena-Molina A, Martínez-López V, et al. Generation of Two Biological Wound Dressings as a Potential Delivery System of Human Adipose-Derived Mesenchymal Stem Cells. ASAIO J. 2015. 61(6): 718-25.

[55] Kucharzewski M, Rojczyk E, Wilemska-Kucharzewska K, Wilk R, Hudecki J, Los MJ. Novel trends in application of stem cells in skin wound healing. Eur J Pharmacol. 2019. 843: 307-315.

[56] Wei Y, Liu Z, Zhu X, et al. Dual directions to address the problem of aseptic loosening via electrospun PLGA @ aspirin nanofiber coatings on titanium. Biomaterials. 2020. 257: 120237.

\section{Figure legend}

Scheme1. LPS/IFN- $\gamma$ activated RAW264.7 cell membrane modified PLGA nanofiber with BMMSCs in promoting diabetic wound healing. The RCM-fibers likely promotes the proliferation of BMMSCs and resistance to oxidative stress in situ. RCM-fiber-BMMSCs thus accelerated wound closure with rapid re-epithelialization, collagen remodeling, immunoregulation, antioxidant stress and angiogenesis in experimental diabetic wound healing in vivo. This is partially achieved through CD200-CD200R axis accompanied by upregulation of Lcn2, TNFAIP6, Lif and Ccl2 in BMMSCs. RCM-fiber-BMMSCs thus function as a living material possessing in situ immune-stimulated capacity mediated by cell membrane to exaggerate the biofunction of BMMSCs.

Figure 1 Physiochemical and biological characterization of RCM-fibers(LPS/IFN- $\gamma$ activated RAW264.7 cell membrane modified (lactic-co-glycolic acid) (PLGA) nanofibers). (A). Representative fluorescent images of RCM-fibers and Un-fibers after coumarin and Dil staining(scale bar $=20 \mu \mathrm{m})$. (B). Detection of $\mathrm{Na}^{+} / \mathrm{K}^{+}$ATPase and CD11c on the RCM-fibers (with LPS/IFN- $\gamma$ activated RAW264.7 cell membrane) and Un-fibers (without LPS/IFN- $\gamma$ activated RAW263.7 cell membrane) by Western blotting. (C). SDS-PAGE analysis of proteins presented on LPS/IFN- $\gamma$ activated RAW264.7 cell membrane and the RCM-fibers. (D). Representative images of the morphology of the RCM-fibers by scanning electron microscopy(scale bar $=10 \mu \mathrm{m}$, 
$5 \mu \mathrm{m})$.(E). Size distribution of the RCM-fibers. (F). Comparisons of water contact angels between the Un-fibers and RCM-fibers. (G). Comparison of the degradation rates between the Un-fibers and RCM-fibers. (H). Fluorescent images of BMMSCs cultured on Un-fibers or RCM-fibers by CFDA-SE staining (scale bar $=50 \mu \mathrm{m}$ ). (I). CCK-8 assays for cell proliferation of BMMSCs on day 1, day 3, day 5 and day 7 . Data were represented as mean $\pm \mathrm{SD}$. Differences were assessed using one-way ANOVA with Tukey's multiple comparison tests. (n $\geq 8) N S$ : non-significant, ${ }^{*} P<$ $0.05, * * P<0.01$

Figure 2 RCM-fiber-BMMSCs accelerate wound closure in diabetic mouse. (A). Experiment designing for diabetes induction followed by wound induction and healing. Mice were fed with a high-fat diet, and diabetes was induced by intraperitoneal injection of streptozocin. Wounds were made after four weeks of diabetes induction, and wound closure was studied for 2 weeks upon the treatment. (B). Representative images of the wounds at day 0, 5, 10 and 15 post-wounding, respectively. (C). Quantification of the residual wounds areas at day 0, 5, 10 and 15, respectively. (D). Representative images of H\&E staining for the wounds at day 15 (left) with high-resolution images of epidemis, dermis and hypodemis (Right) (scale bar $=1 \mathrm{~mm}, 50 \mu \mathrm{m})$. (E). Quantification of average epidermal thickness of the wounds. Data represented as mean \pm SD. Differences were assessed using two-way analysis of variance (ANOVA) with Tukey's multiple comparison tests and one-way ANOVA with Tukey's multiple comparison tests. ( $\mathrm{n} \geq 8) N S$ : non-significant, $* P<0.05, * * P<$ $0.01)$.

Figure 3 RCM-fiber-BMMSCs remodel collagen deposition at the wound areas. (A). Masson's trichrome staining for tissue sections (Left) with high-resolution images of collagen deposition in the epidemis, dermis and hypodemis (Right) in diabetic mouse wounds (scale bar $=1 \mathrm{~mm}, 50 \mu \mathrm{m}$ ). (B). Picrosirius red staining for collagen deposition and remodelling in the wounds from diabetic mice (scale bar $=50 \mu \mathrm{m})$. (C). Comparisons of collagen 1 and collagen 3 deposition among different groups. (D). Immunostaining for 8-OHdG (i), CK10 (ii) and CD31 (iii) in wound tissues upon different treatment (scale bar $=50 \mu \mathrm{m})$. Data represented as mean \pm SD. Differences were analysed by one-way ANOVA followed by Tukey's multiple comparison test. ( $\mathrm{n}$ 2 8) NS: non-significant, $* P<0.05, * * P<0.01$

Figure 4 RCM-fiber-BMMSCs reduce the inflammation in the wound areas of 
diabetic mice. (A). Immunofluorescence staining for F4/80 (red), CD206 (green), CD86 (green) and TNF- $\alpha$ (green) in wound tissues (scale bar $=50 \mu \mathrm{m})$. (B). Comparisons of the proportions of $\mathrm{F} 4 / 80^{+} / \mathrm{CD} 86^{+}, \mathrm{F} 4 / 80^{+} / \mathrm{TNF}-\alpha^{+}$and $\mathrm{F} 4 / 80^{+} / \mathrm{CD} 206^{+}$ cells in the wound areas among different groups. (C-D). Expression levels of IL-1 $\beta$ (C) and IL-10 (D) in diabietic cutaneous wound on day 7 after wounding by real-time PCR. (E). Relative expression levels of Ccl2, Lif, TNFAIP6 and Lcn2 in the diabetic skin wounds treated with different therapeutic means. Data represented as mean $\pm \mathrm{SD}$. Differences were analyzed using one-way ANOVA followed by Tukey's multiple comparison test. ( $\mathrm{n} \geq 8) N S$ : non-significant, $* P<0.05,{ }^{*} * P<0.01$.

Figure 5 RCM-fibers promote wound healing effects of BMMSCs in vitro. (A). Schematic illustration showing the cocultures of BMMSCs with the RCM-fibers in vitro. (B). Gene ontology enrichment scatter plots in key signaling pathways. (C). Heat map analysis of wound healing related gene expression in BMMSCs coculturing with either the Un-fibers or the RCM-fibers for 48 h. (D). Validation of expression levels of CD200, Ccl2, Lif, TNFAIP6 and Lcn2 in BMMSCs coculturing with either the Un-fibers or the RCM-fibers for $48 \mathrm{~h}$ by real-time PCR. (E). Schematic illustration on the migration of mouse epithelial cell line JB6 incubated with either RCM-fiber-BMMSCs or Un-fiber-BMMSCs upon $\mathrm{H}_{2} \mathrm{O}_{2}$ treatment. (F). The representative images of wound areas covered by JB6 cell at 12, 18, 24 and $36 \mathrm{~h}$ $($ scale bar $=50 \mu \mathrm{m}) .(\mathbf{G})$. Comparisons of normalized wound areas covered by JB6 cell at different time points. (H). CD86 and CD206 expressions on LPS/IFN- $\gamma$ activated RAW264.7 cells after incubating with RCM-fiber-BMMSCs or Un-fiber-BMMSCs. (I). TNF- $\alpha$ expression in LPS/IFN- $\gamma$ activated RAW264.7 cells after incubating with the RCM-fiber-BMMSCs or Un-fiber-BMMSCs. Data were represented as mean \pm SD. Differences were analyzed by one-way ANOVA with Tukey's multiple comparison test. The experiments were repeated in triplicates. ${ }^{*} P<0.05,{ }^{*} * P<0.01$

Figure 6 CD200-CD200R axis is involved in promoting healing effects of RCMfiber-BMMSCs in vivo. (A). Representative images of the wound areas at day 0, 5, 10 and 15 post-wounding upon anti-CD200R or isotype IgG treatment. (B).

Representative images of $\mathrm{H} \& \mathrm{E}$ staining for the wounds at day 15 (left) with highresolution images of epidemis, dermis and hypodemis (Right) upon anti-CD200R or isotype $\mathrm{IgG}$ treatment (scale bar $=1 \mathrm{~mm}, 50 \mu \mathrm{m}$ ). (C). Comparisons of the residual wound areas at each time point upon anti-CD200R or isotype IgG treatment. (D). Comparisons of average epidermal thickness of the wounds upon anti-CD200R or isotype IgG treatment. (E). Images of Masson's trichrome staining for wound tissues at day 15 (blue for collagen) (scale bar $=1 \mathrm{~mm}, 50 \mu \mathrm{m})$. (F). Representative images of 
wound areas at day 0, 5, 10 and 15 post-wounding upon RCM-fiber-BMMSCs treatment with or without CD200 expressions in BMMSCs. (G). Representative images of H\&E staining for the wounds at day 15 (left) with high-resolution images of epidemis, dermis and hypodemis (Right) upon RCM-fiber-BMMSCs treatment with or without $C D 200$ expressions in BMMSCs(scale bar $=1 \mathrm{~mm}, 50 \mu \mathrm{m})$. (H-I). Comparisons of the residual wound areas at each time point $(\mathrm{H})$ and average epidermal thickness of the wounds (I) upon RCM-fiber-BMMSCs treatment with or without CD200 expressions in BMMSCs. (J). Images of Masson's trichrome staining for wound tissues at day 15 (blue for collagen) upon RCM-fiber-BMMSCs treatment with or without $C D 200$ expressions in BMMSCs(scale bar $=1 \mathrm{~mm}, 50 \mu \mathrm{m})$. (K). Picrosirius red staining for collagen deposition in the wound areas(scale bar $=50 \mu \mathrm{m})$. (L). Comparison of the proportions of Collagen 1 and Collagen 3 depositing in diabetic wounds upon CD200 interference in BMMSCs. (M). The levels of TNFAIP6 in wound tissues upon RCM-fiber-BMMSCs treatment with or without CD200 expressions in BMMSCs. Data were represented as mean \pm SD. Differences were analyzed through one-way ANOVA with Tukey's multiple comparison test. ( $\mathrm{n} \geq 8)$ NS: non-significant, $* P<0.05, * * P<0.01$

Figure 7 Physiochemical and biological characterization of TCM-fibers(LPS/IFN- $\gamma$ activated human THP-1 cell membrane modified(lactic-co-glycolic acid) (PLGA) nanofibers). (A). Representative fluorescent images of the TCM-fibers and Un-fibers after coumarin and Dil staining(scale bar $=20 \mu \mathrm{m}) . \quad$ (B). Detection of $\mathrm{Na}^{+} / \mathrm{K}^{+}$ ATPase and CD11c on the TCM-fibers and Un-fibers (without LPS/IFN- $\gamma$ activated RAW263.7 cell membrane) by Western blotting. (C). Representative images of the morphology of the TCM-fibers by scanning electron microscopy (scale bar $=10 \mu \mathrm{m}$, $5 \mu \mathrm{m}$ ). (D). Size distribution of the TCM-fibers. (E). Fluorescent images of BMMSCs cultured on Un-fibers or RCM-fibers by CFDA-SE staining(scale bar $=5 \mu \mathrm{m})$. (F). CCK-8 assays for cell proliferation of BMMSCs on day 1, day 3, day 5 and day 7. (G). The representative images of wound areas covered by HACAT cells at 12, 18, 24 and $36 \mathrm{~h}($ scale bar $=25 \mu \mathrm{m})$. (H). Comparisons of normalized wound areas covered by HACAT cells at different time points. Data were represented as mean \pm SD. Differences were analyzed by one-way ANOVA with Tukey's multiple comparison tests. $(\mathrm{n} \geq 3) * P<0.05, * * P<0.01$

Figure 8 TCM-fibers-hBMMSCs improve wound healing in diabetic wound. (A). Representative images of the wounds at day $0,5,10$ and 15 post-wounding, respectively. (B). Quantification of the residual wounds areas at day 0, 5, 10 and 15, 
respectively. (C). Representative images of H\&E staining for the wounds at day 15 (left) with high-resolution images of epidemis, dermis and hypodemis (Right)(scale bar $=1 \mathrm{~mm}, 50 \mu \mathrm{m})$. (D). Quantification of average epidermal thickness of the wounds. (E). Masson's trichrome staining for tissue sections (Left) with high-resolution images of collagen deposition in the epidemis, dermis and hypodemis (Right) in diabetic mouse wounds(scale bar $=1 \mathrm{~mm}, 50 \mu \mathrm{m})$. (F). Picrosirius red staining for collagen deposition and remodelling in the wounds from diabetic mice(scale bar $=50 \mu \mathrm{m})$. (G). Comparisons of collagen 1 and collagen 3 deposition among different groups. Data were represented as mean $\pm \mathrm{SD}$. Differences were analized through two-way and oneway ANOVA with Tukey's multiple comparison tests. $(\mathrm{n} \geq 8) N S$ : non-significant, ${ }^{*} P$ $<0.05, * * P<0.01$ 
A

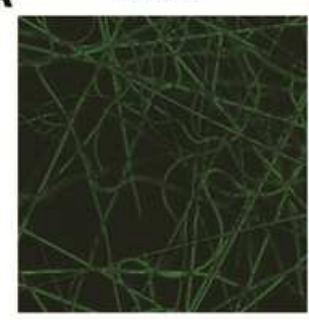

RCM-fiber

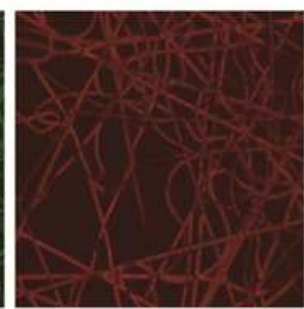

B

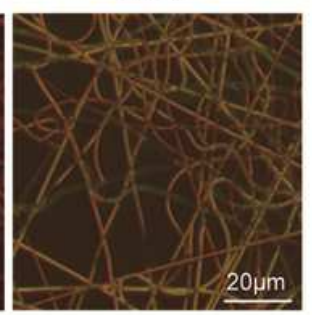

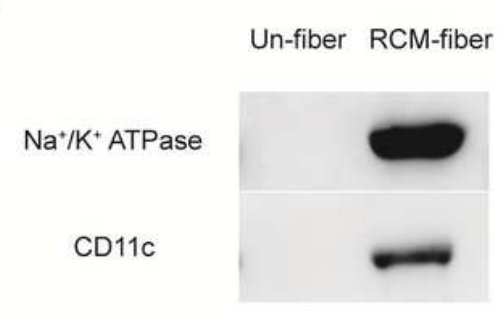

E

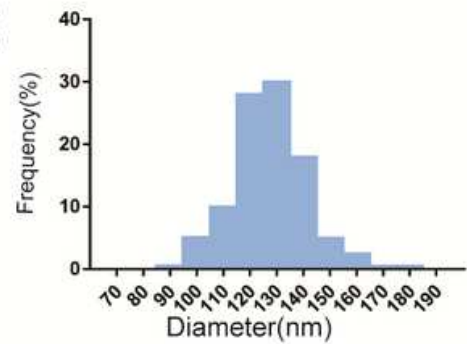

G

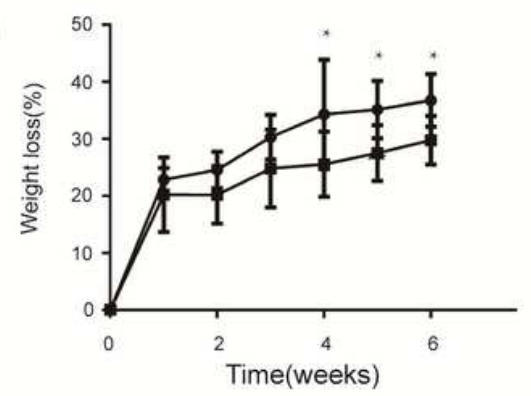

C

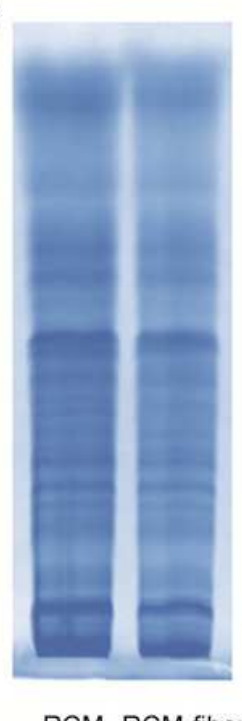

RCM RCM-fiber

$\rightarrow$ RCM-fiber

- Un-fiber

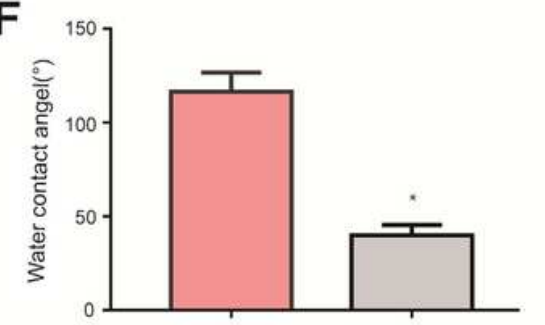

$\square$ Un-fiber

$\square$ RCM-fiber

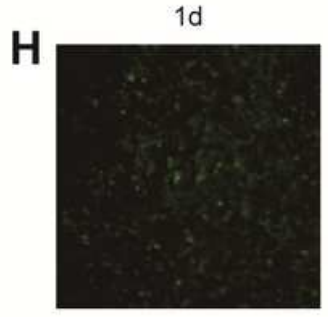

$4 d$

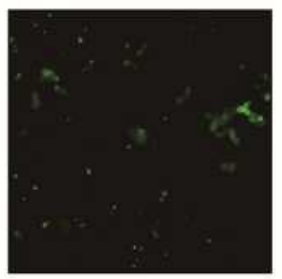

$7 d$
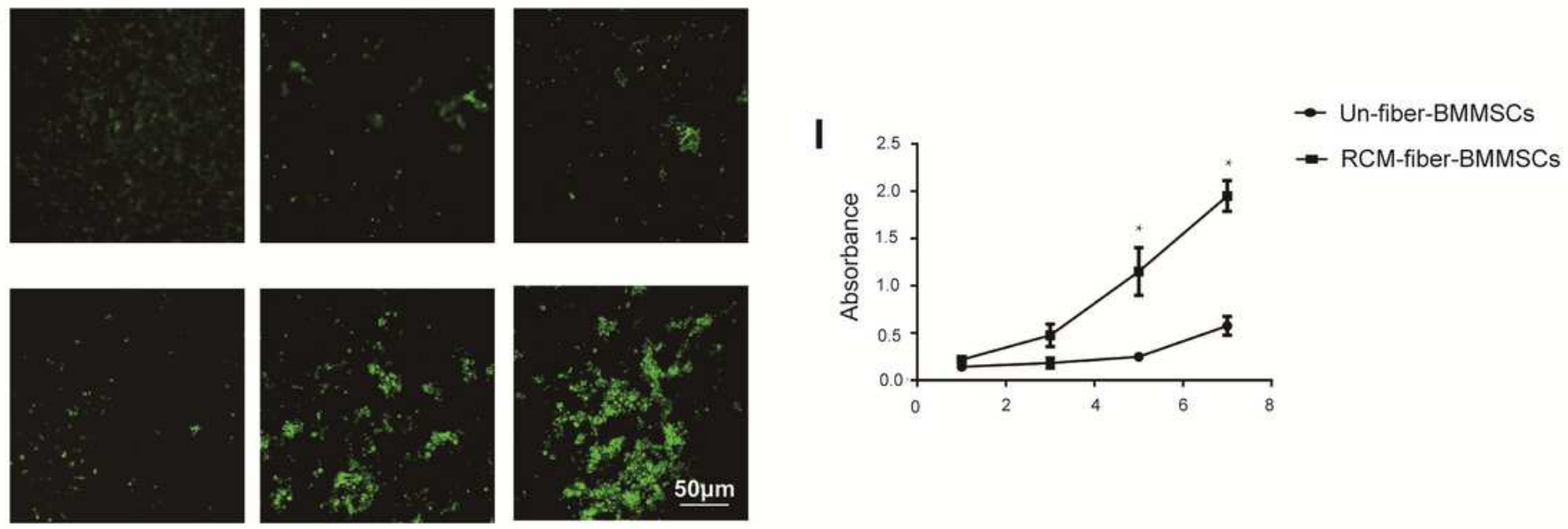

\section{Figure 1}

Physiochemical and biological characterization of RCM-fibers(LPS/IFN- $y$ activated RAW264.7 cell membrane modified (lactic-co-glycolic acid) (PLGA) nanofibers). (A). Representative fluorescent images of RCM-fibers and Un-fibers after coumarin and Dil staining(scale bar $=20 \mu \mathrm{m}$ ). (B). Detection of $\mathrm{Na}+/ \mathrm{K}+$ ATPase and CD11c on the RCM-fibers (with LPS/IFN- $y$ activated RAW264.7 cell membrane) and Un-fibers (without LPS/IFN- $y$ activated RAW263.7 cell membrane) by Western blotting. (C). SDS-PAGE analysis of 
proteins presented on LPS/IFN- $y$ activated RAW264.7 cell membrane and the RCM-fibers. (D).

Representative images of the morphology of the RCM-fibers by scanning electron microscopy (scale bar = $10 \mu \mathrm{m}$,

$5 \mu \mathrm{m})$.(E). Size distribution of the RCM-fibers. (F). Comparisons of water contact angels between the Unfibers and RCM-fibers. (G). Comparison of the degradation rates between the Un-fibers and RCM-fibers. $(\mathrm{H})$. Fluorescent images of BMMSCs cultured on Un-fibers or RCM-fibers by CFDA-SE staining (scale bar = $50 \mu \mathrm{m})$. (I). CCK-8 assays for cell proliferation of BMMSCs on day 1, day 3, day 5 and day 7. Data were represented as mean \pm SD. Differences were assessed using one-way ANOVA with Tukey's multiple comparison tests. $(n \geq 8)$ NS: non-significant, $* P<0.05,{ }^{*} \mathrm{P}<0.01$.

A
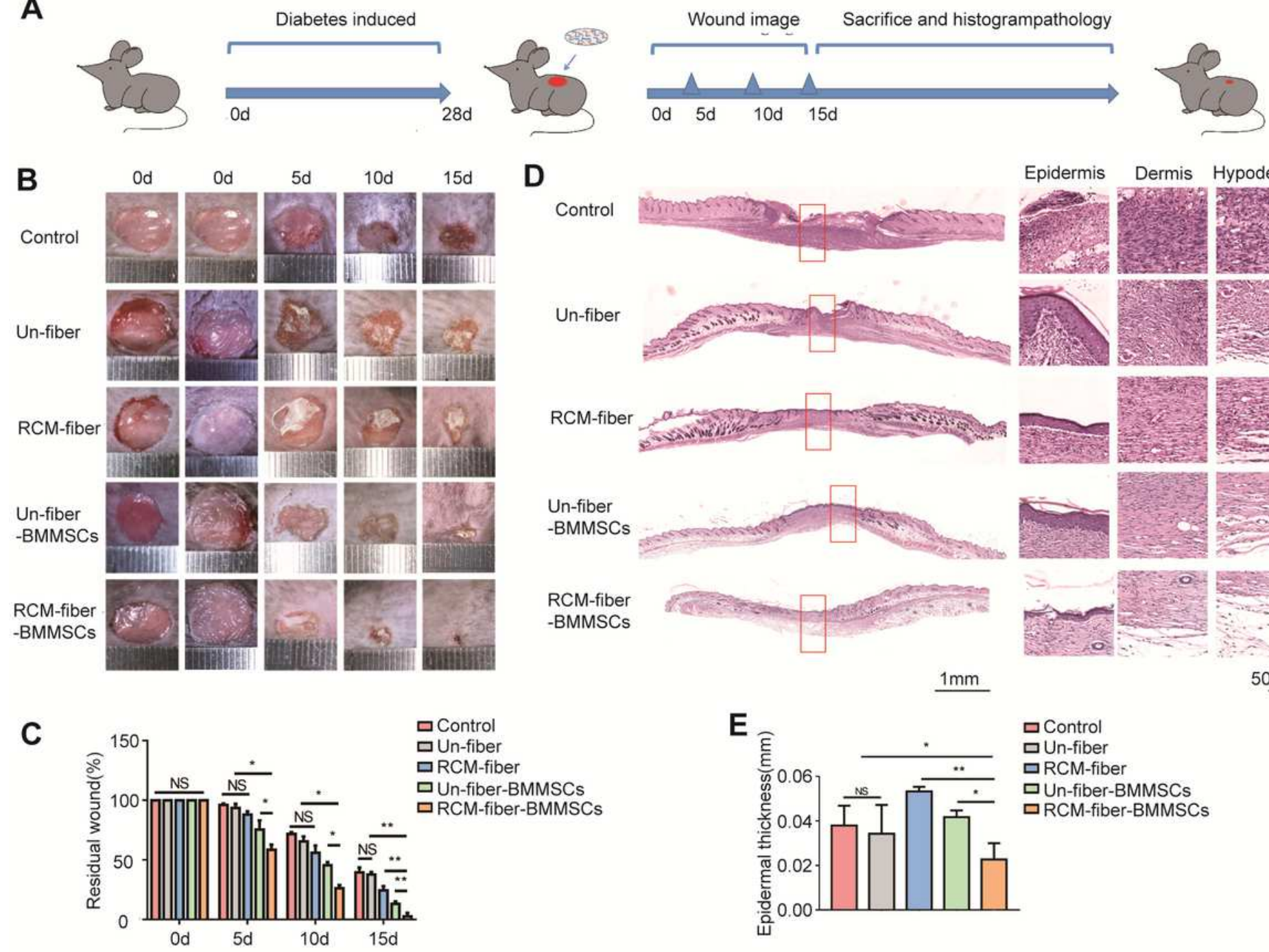

Epidermis

Dermis Hypodermis
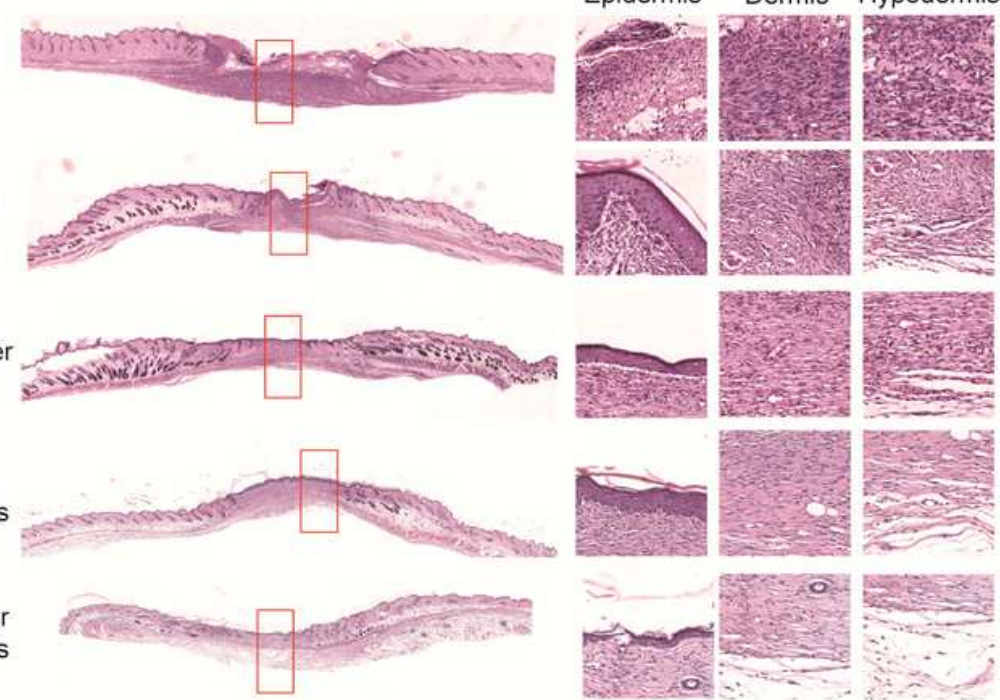

Un-fiber
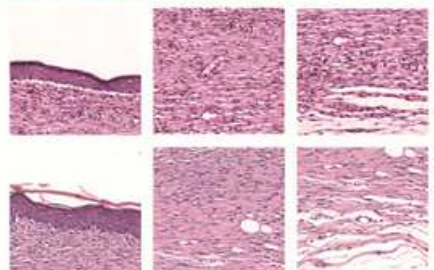

RCM-fiber
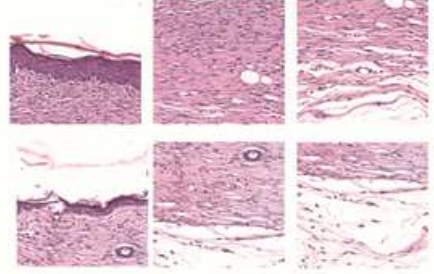

$1 \mathrm{~mm}$

$50 \underline{\mu m}$

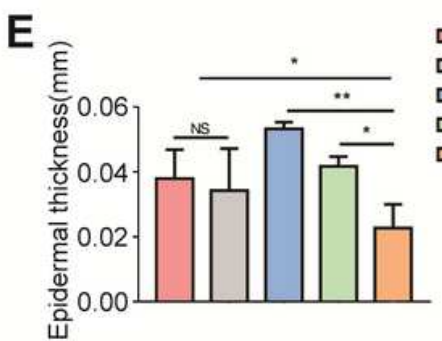

$\square$ Control

口Un-fiber

ㅁ RCM-fiber

Un-fiber-BMMSCs

$\square$ RCM-fiber-BMMSCs

\section{Figure 2}

RCM-fiber-BMMSCs accelerate wound closure in diabetic mouse. (A). Experiment designing for diabetes induction followed by wound induction and healing. Mice were fed with a high-fat diet, and diabetes was induced by intraperitoneal injection of streptozocin. Wounds were made after four weeks of diabetes induction, and wound closure was studied for 2 weeks upon the treatment. (B). Representative images of 
the wounds at day 0, 5, 10 and 15 post-wounding, respectively. (C). Quantification of the residual wounds areas at day $0,5,10$ and 15, respectively. (D). Representative images of H\&E staining for the wounds at day 15 (left) with high-resolution images of epidemis, dermis and hypodemis (Right) (scale bar $=1 \mathrm{~mm}$, $50 \mu \mathrm{m})$. (E). Quantification of average epidermal thickness of the wounds. Data represented as mean \pm SD. Differences were assessed using two-way analysis of variance (ANOVA) with Tukey's multiple comparison tests and one-way ANOVA with Tukey's multiple comparison tests. $(n \geq 8)$ NS: nonsignificant, $* \mathrm{P}<0.05, \star \star \mathrm{P}<0.01)$.

A

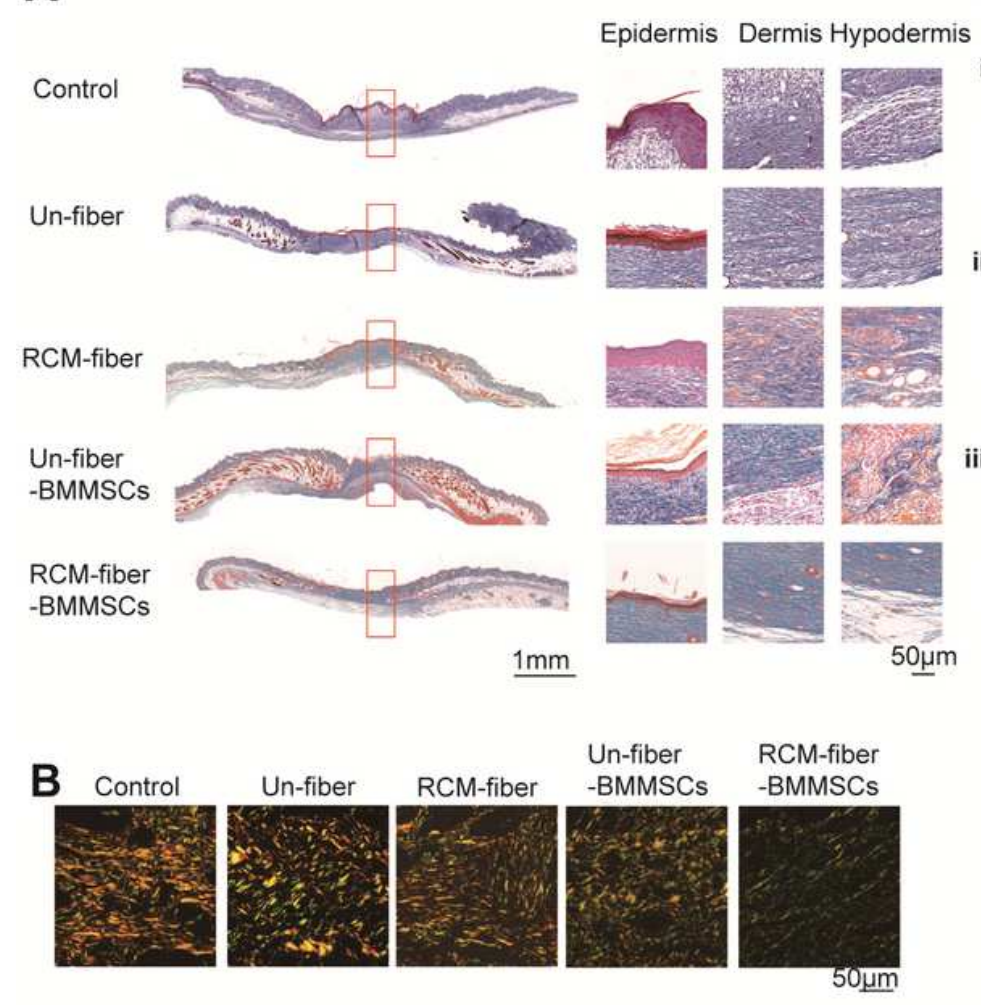

D
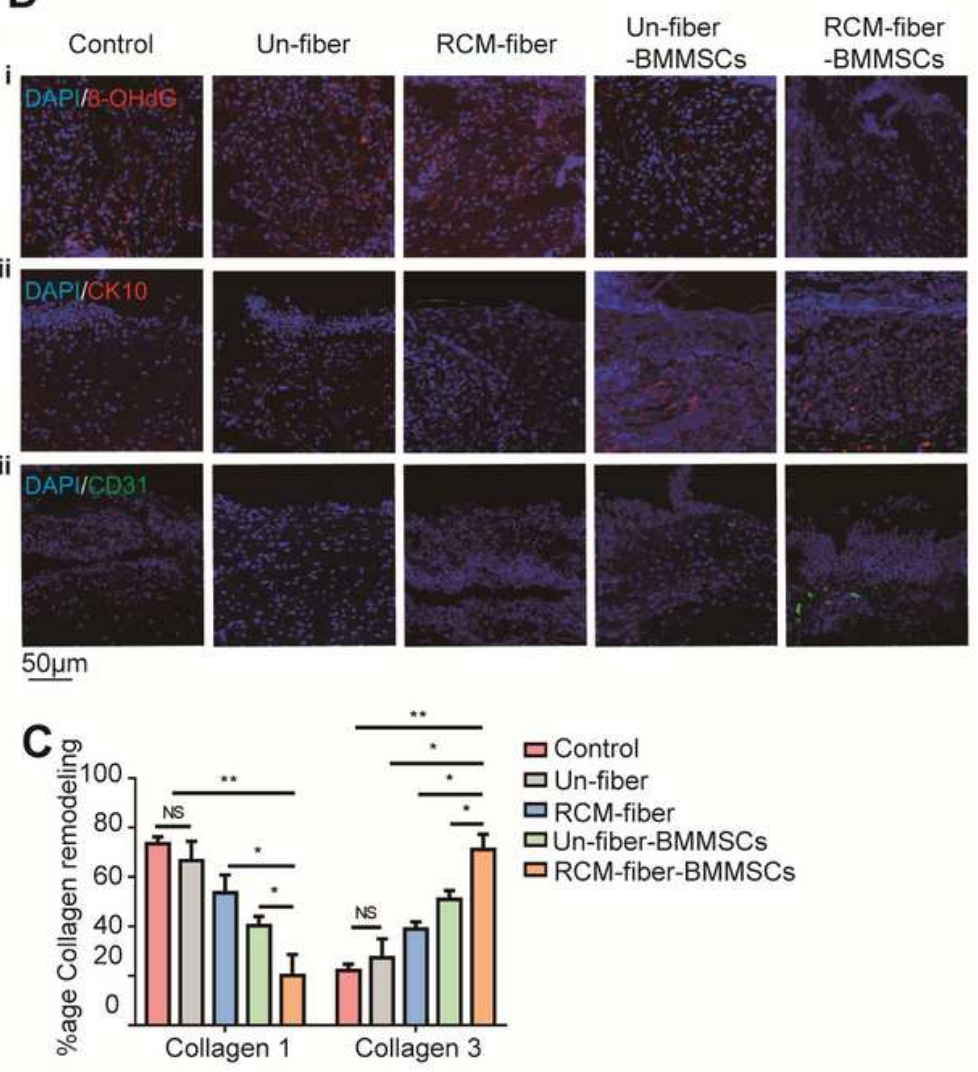

\section{Figure 3}

RCM-fiber-BMMSCs remodel collagen deposition at the wound areas. (A). Masson's trichrome staining for tissue sections (Left) with high-resolution images of collagen deposition in the epidemis, dermis and hypodemis (Right) in diabetic mouse wounds (scale bar $=1 \mathrm{~mm}, 50 \mu \mathrm{m})$. (B). Picrosirius red staining for collagen deposition and remodelling in the wounds from diabetic mice (scale bar=50 $\mu \mathrm{m}$ ). (C). Comparisons of collagen 1 and collagen 3 deposition among different groups. (D). Immunostaining for 8OHdG (i), CK10 (ii) and CD31 (iii) in wound tissues upon different treatment (scale bar $=50 \mu \mathrm{m}$ ). Data represented as mean $\pm S D$. Differences were analysed by one-way ANOVA followed by Tukey's multiple comparison test. $(n \geq 8)$ NS: non-significant, ${ }^{*} P<0.05,{ }^{*} \mathrm{P}<0.01$ 
A
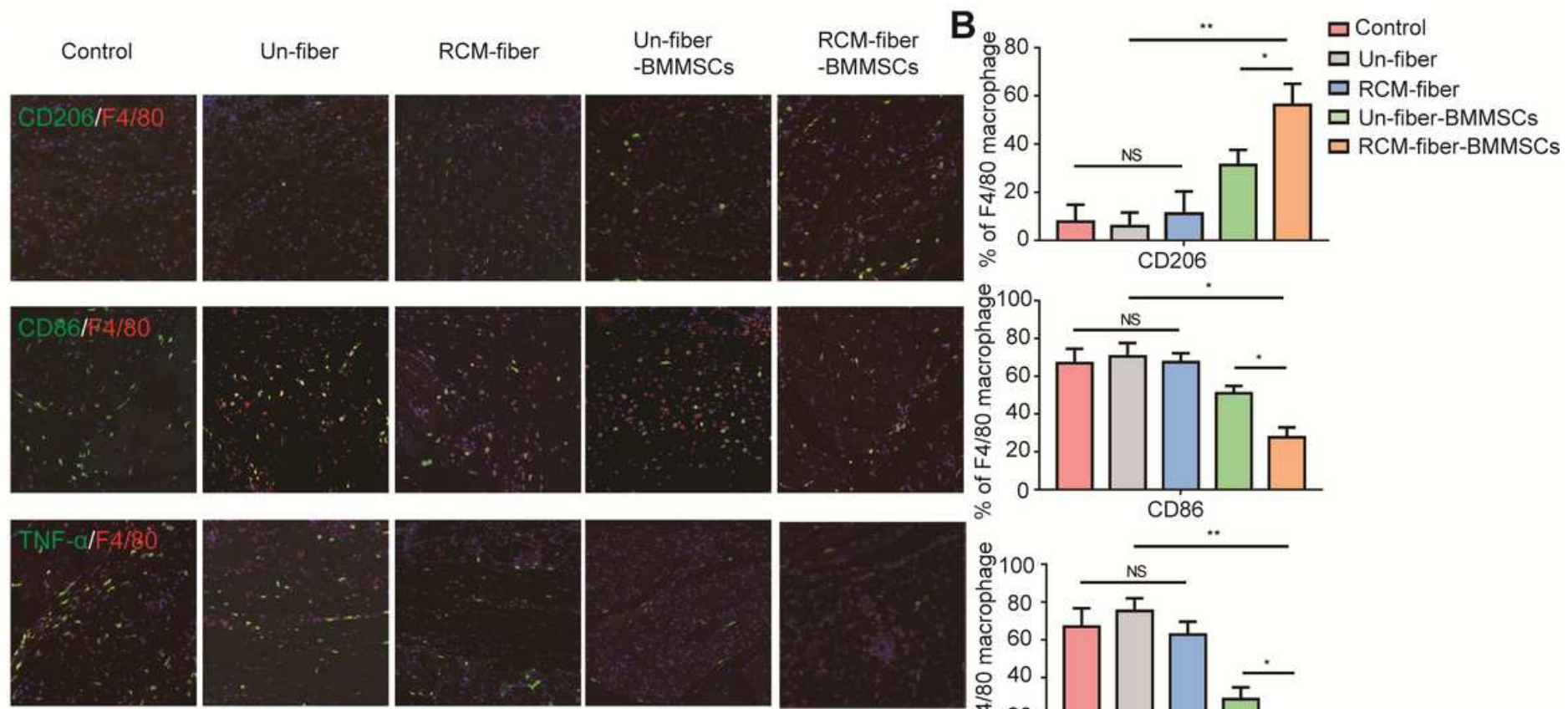

$5 \underline{\underline{5 m}}$
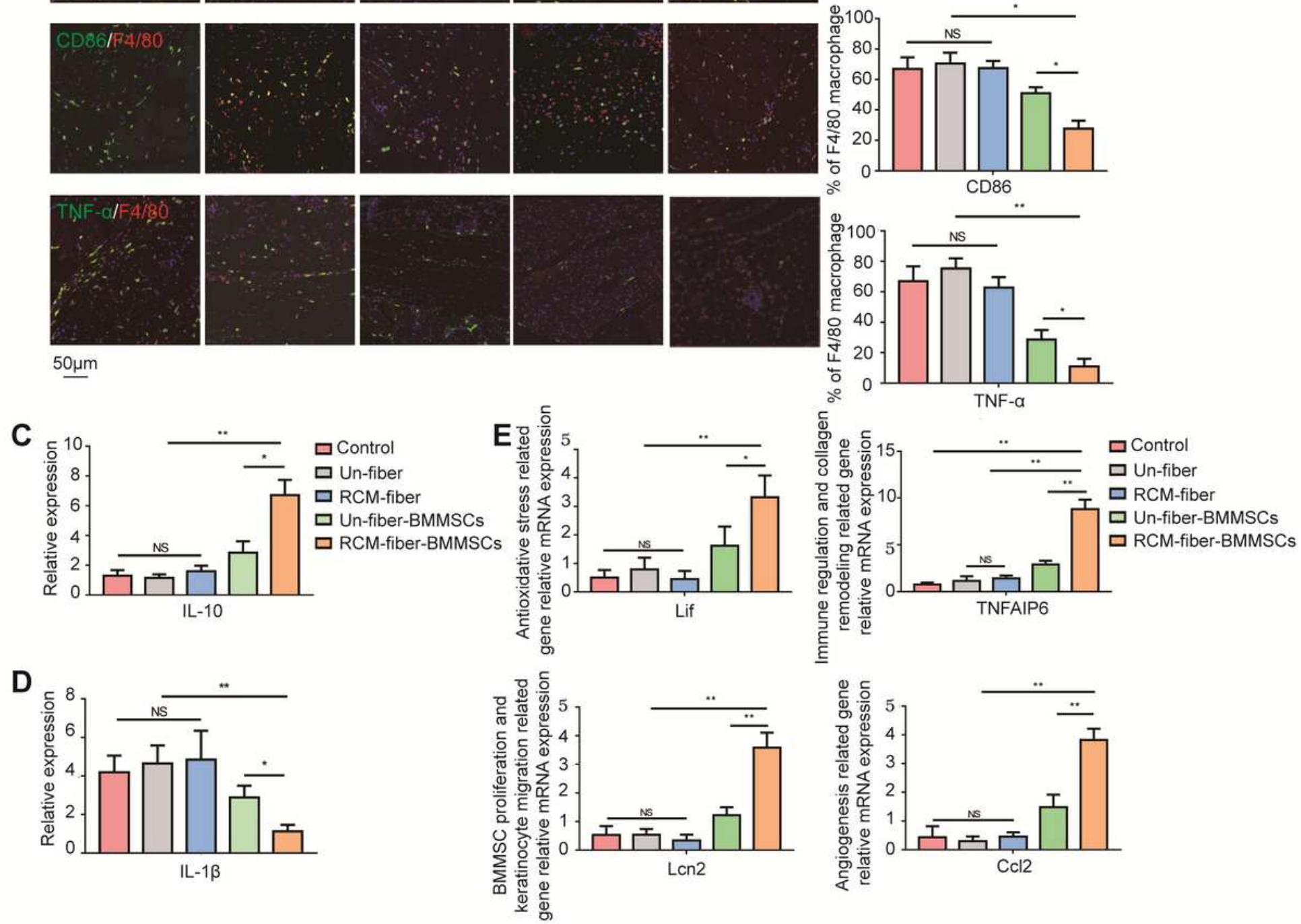

\section{Figure 4}

RCM-fiber-BMMSCs reduce the inflammation in the wound areas of diabetic mice. (A). Immunofluorescence staining for F4/80 (red), CD206 (green), CD86 (green) and TNF-a (green) in wound tissues (scale bar $=50 \mu \mathrm{m})$. (B). Comparisons of the proportions of F4/80+/CD86+, F4/80+/TNF- $\mathrm{a}+$ and $\mathrm{F} 4 / 80+/ \mathrm{CD} 206+$ cells in the wound areas among different groups. (C-D). Expression levels of IL-1 $\beta$ (C) and IL-10 (D) in diabietic cutaneous wound on day 7 after wounding by real-time PCR. (E). Relative expression levels of Ccl2, Lif, TNFAIP6 and Lcn2 in the diabetic skin wounds treated with different therapeutic means. Data represented as mean \pm SD. Differences were analyzed using one-way ANOVA followed by Tukey's multiple comparison test. $(n \geq 8)$ NS: non-significant, ${ }^{*} P<0.05,{ }^{\star} \mathrm{P}<0.01$. 

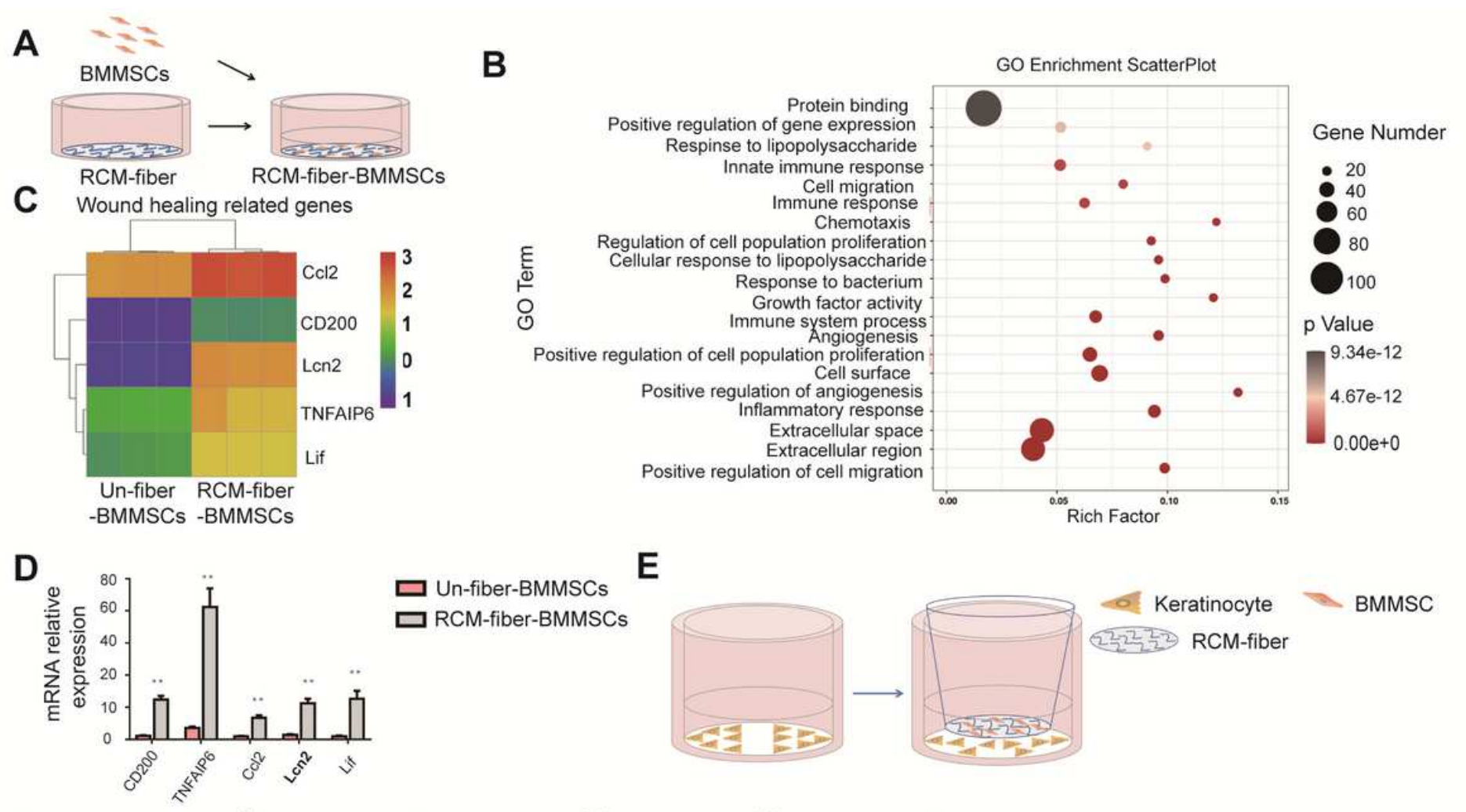

E

$\mathbf{F}$

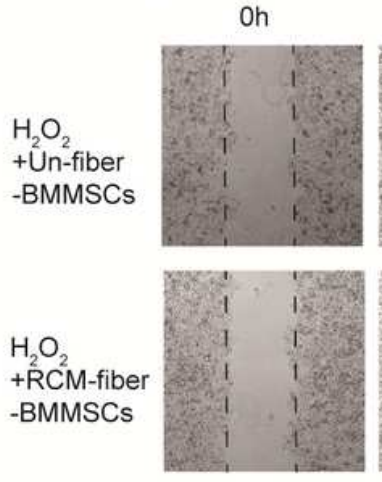

H

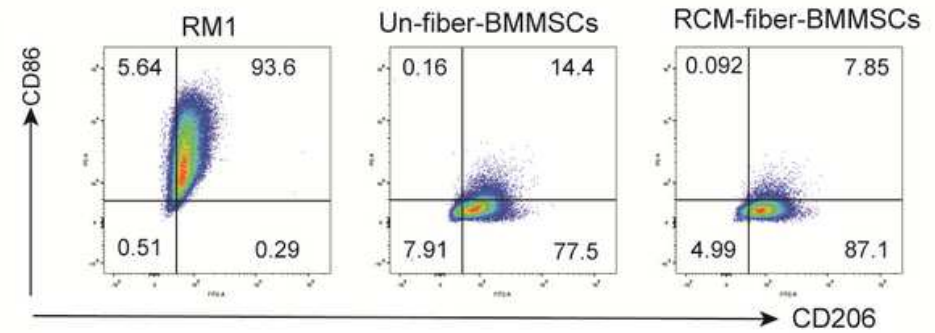

$12 \mathrm{~h}$
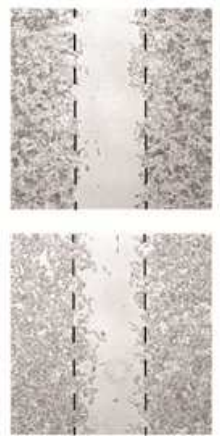

$24 \mathrm{~h}$

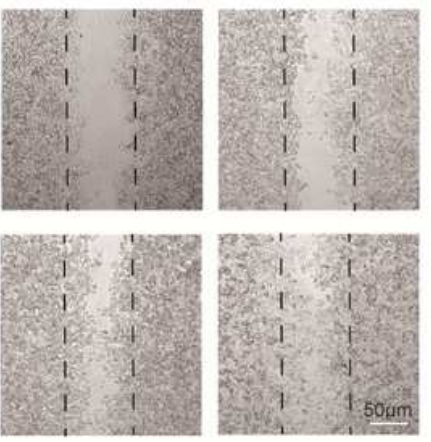

G

BMMSC

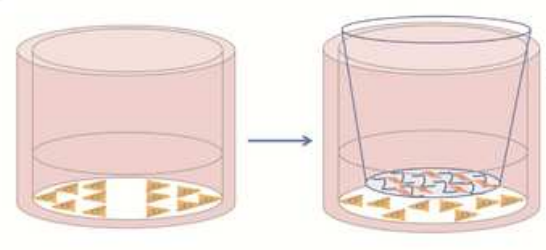

6 Keratinocyte

$2_{2}^{2}-22$ RCM-fiber

\section{G}
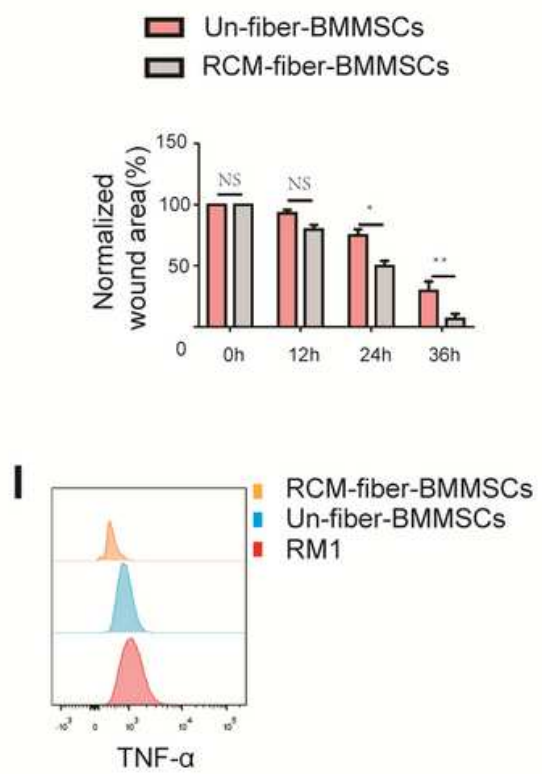

\section{Figure 5}

RCM-fibers promote wound healing effects of BMMSCs in vitro. (A). Schematic illustration showing the cocultures of BMMSCs with the RCM-fibers in vitro. (B). Gene ontology enrichment scatter plots in key signaling pathways. (C). Heat map analysis of wound healing related gene expression in BMMSCs coculturing with either the Un-fibers or the RCM-fibers for $48 \mathrm{~h}$. (D). Validation of expression levels of CD200, Ccl2, Lif, TNFAIP6 and Lcn2 in BMMSCs coculturing with either the Un-fibers or the RCM-fibers for $48 \mathrm{~h}$ by real-time PCR. (E). Schematic illustration on the migration of mouse epithelial cell line JB6 incubated with either RCM-fiber-BMMSCs or Un-fiber-BMMSCs upon $\mathrm{H} 2 \mathrm{O} 2$ treatment. $(F)$. The 
representative images of wound areas covered by JB6 cell at 12, 18, 24 and $36 \mathrm{~h}$ (scale bar $=50 \mu \mathrm{m}$ ). (G). Comparisons of normalized wound areas covered by JB6 cell at different time points. (H). CD86 and CD206 expressions on LPS/IFN-y activated RAW264.7 cells after incubating with RCM-fiber-BMMSCs or Un-fiber-BMMSCs. (I). TNF- $a$ expression in LPS/IFN- $\gamma$ activated RAW264.7 cells after incubating with the RCM-fiber-BMMSCs or Un-fiber-BMMSCs. Data were represented as mean \pm SD. Differences were analyzed by one-way ANOVA with Tukey's multiple comparison test. The experiments were repeated in triplicates. ${ }^{*} \mathrm{P}<0.05,{ }^{*} \mathrm{P}<0.01$

A

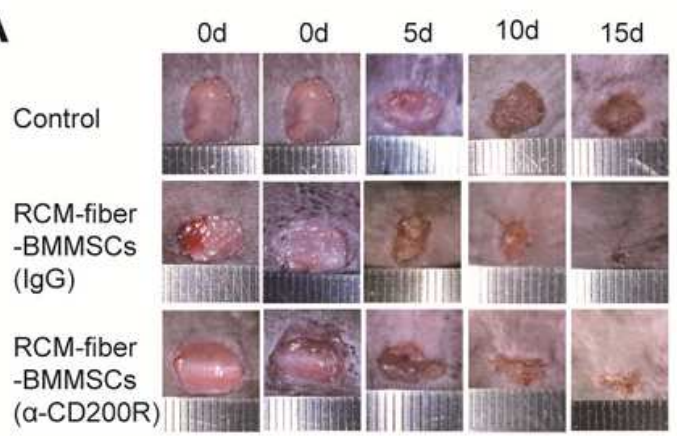

B

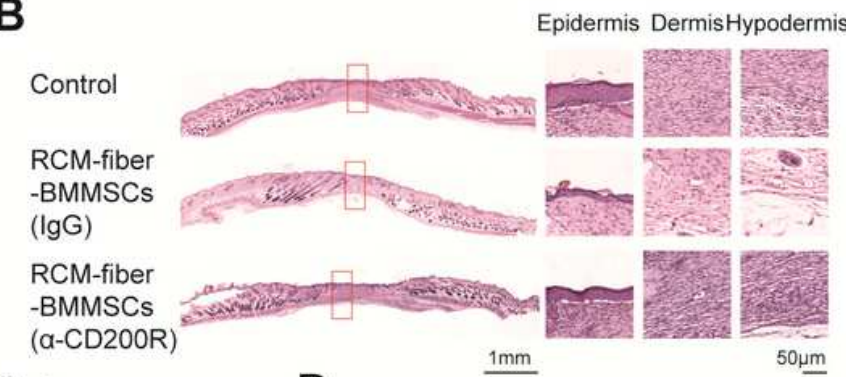

C

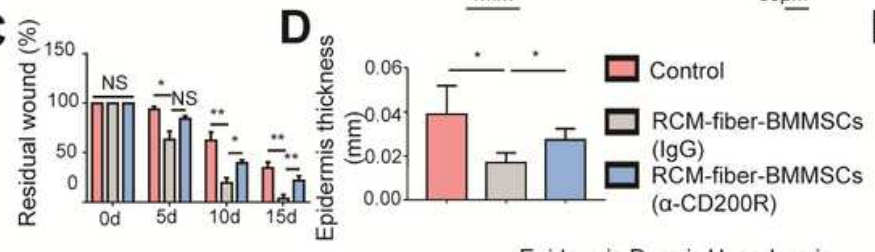

E

Control

RCM-fiber

-BMMSCs

(lgG)

RCM-fiber

-BMMSCs

(a-CD200R)

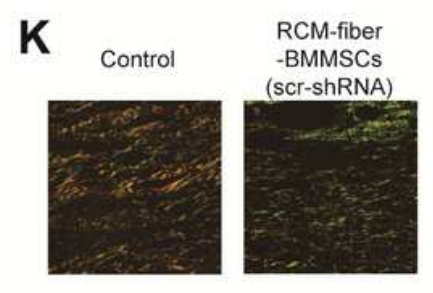
Epidermis Dermis Hypoderm is

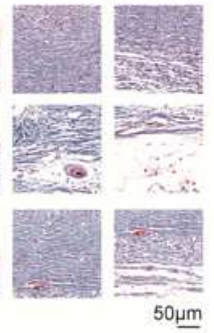

-BMMSCs L (CD200-shRNA)

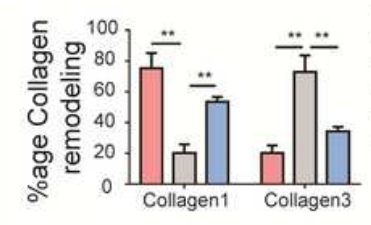

F

Control

RCM-fiber

-BMMSCs

(scr-shRNA)

RCM-fiber

-BMMSCs

(CD200-shRNA)
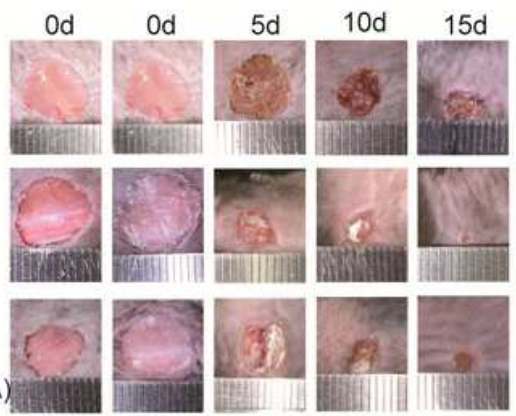

G

Control

RCM-fiber -BMMSCs (scr-shRNA)

RCM-fiber

-BMMSCs (CD200-shRNA)
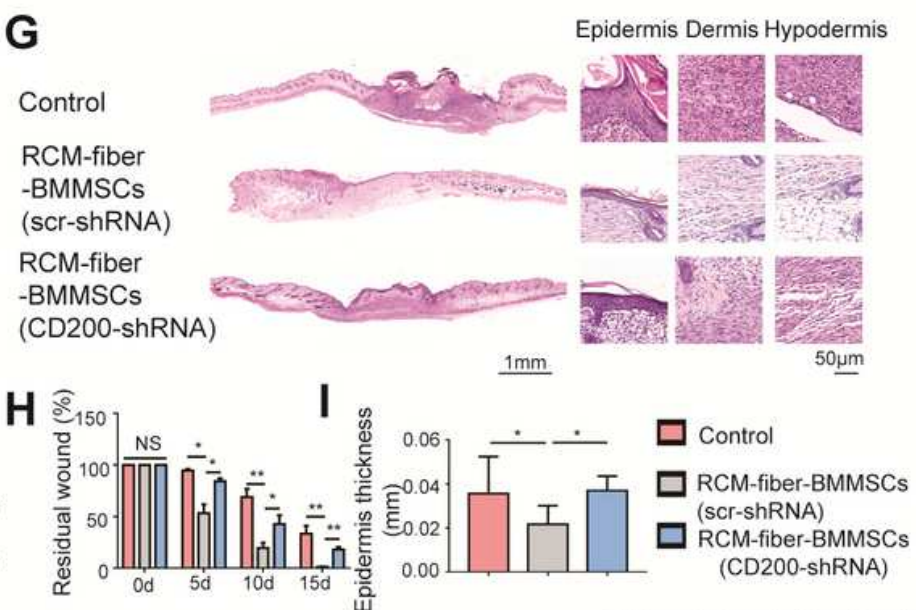

J

Control

RCM-fiber -BMMSCs (scr-shRNA)

RCM-fiber

-BMMSCs

(CD200-shRNA)

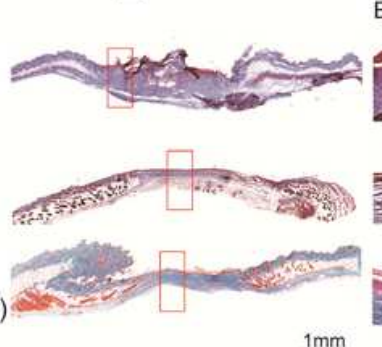

Epidermis Dermis Hypodermis
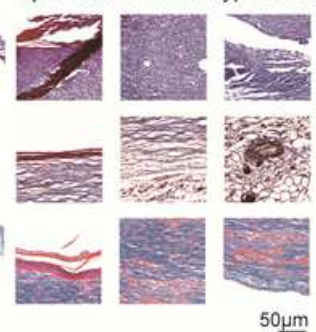

$1 \mathrm{~mm}$

M

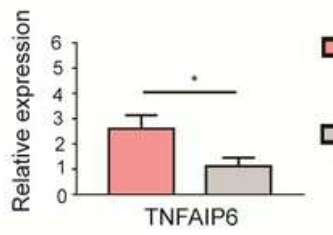

RCM-fiber-BMMSCs (scr-shRNA)

RCM-fiber-BMMSCs (CD200-shRNA)

Figure 6 
CD200-CD200R axis is involved in promoting healing effects of RCMfiber- BMMSCs in vivo. (A).

Representative images of the wound areas at day 0, 5, 10 and 15 post-wounding upon anti-CD200R or isotype IgG treatment. (B). Representative images of H\&E staining for the wounds at day 15 (left) with highresolution images of epidemis, dermis and hypodemis (Right) upon anti-CD200R or isotype IgG treatment (scale bar $=1 \mathrm{~mm}, 50 \mu \mathrm{m}$ ). (C). Comparisons of the residual wound areas at each time point upon anti-CD200R or isotype $\lg G$ treatment. (D).

Comparisons of average epidermal thickness of the wounds upon anti-CD200R or isotype IgG treatment. (E). Images of Masson's trichrome staining for wound tissues at day 15 (blue for collagen) (scale bar = $1 \mathrm{~mm}, 50 \mu \mathrm{m})$. (F). Representative images of wound areas at day $0,5,10$ and 15 post-wounding upon RCM-fiber-BMMSCs treatment with or without CD200 expressions in BMMSCs. (G). Representative images of H\&E staining for the wounds at day 15 (left) with high-resolution images of epidemis, dermis and hypodemis (Right) upon RCM-fiber-BMMSCs treatment with or without CD200 expressions in BMMSCs(scale bar $=1 \mathrm{~mm}, 50 \mu \mathrm{m}) .(\mathrm{H}-\mathrm{I})$. Comparisons of the residual wound areas at each time point $(\mathrm{H})$ and average

epidermal thickness of the wounds (I) upon RCM-fiber-BMMSCs treatment with or without CD200 expressions in BMMSCs. $(\mathrm{J})$. Images of Masson's trichrome staining for wound tissues at day 15 (blue for collagen) upon RCM-fiber-BMMSCs treatment with or without CD200 expressions in BMMSCs(scale bar $=1 \mathrm{~mm}, 50 \mu \mathrm{m}) .(\mathrm{K})$. Picrosirius red staining for collagen deposition in the wound areas(scale bar $=$ $50 \mu \mathrm{m})$. (L). Comparison of the proportions of Collagen 1 and Collagen 3 depositing in diabetic wounds upon CD200 interference in BMMSCs. (M). The levels of TNFAIP6 in wound tissues upon RCM-fiberBMMSCs treatment with or without CD200 expressions in BMMSCs. Data were represented as mean \pm SD. Differences were analyzed through one-way ANOVA with Tukey's multiple comparison test. $(n \geq 8)$ NS: non-significant, ${ }^{*} P<0.05, * * P<0.01$ 
A

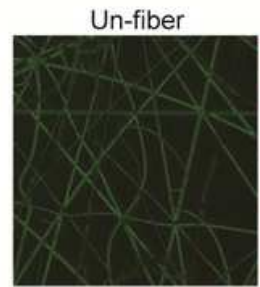

TCM-fiber

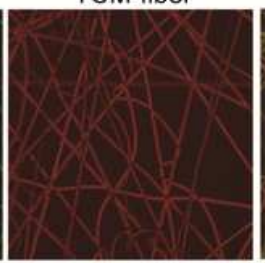

C

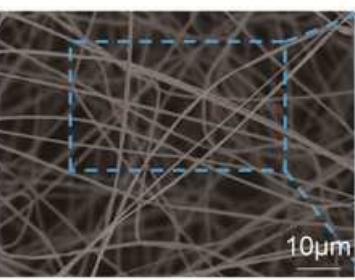

Merge

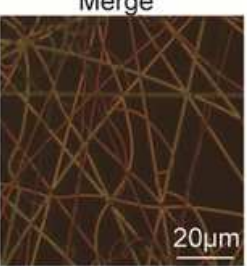

B
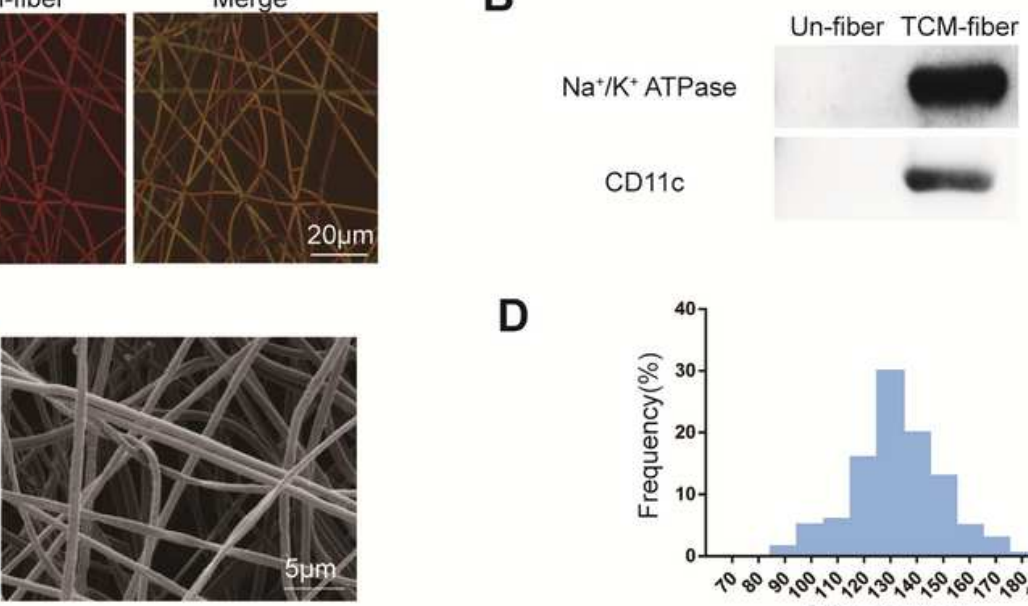

D

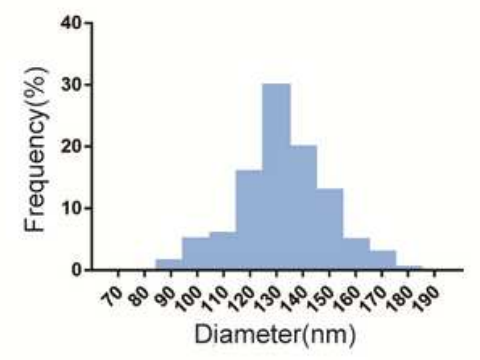

Oh
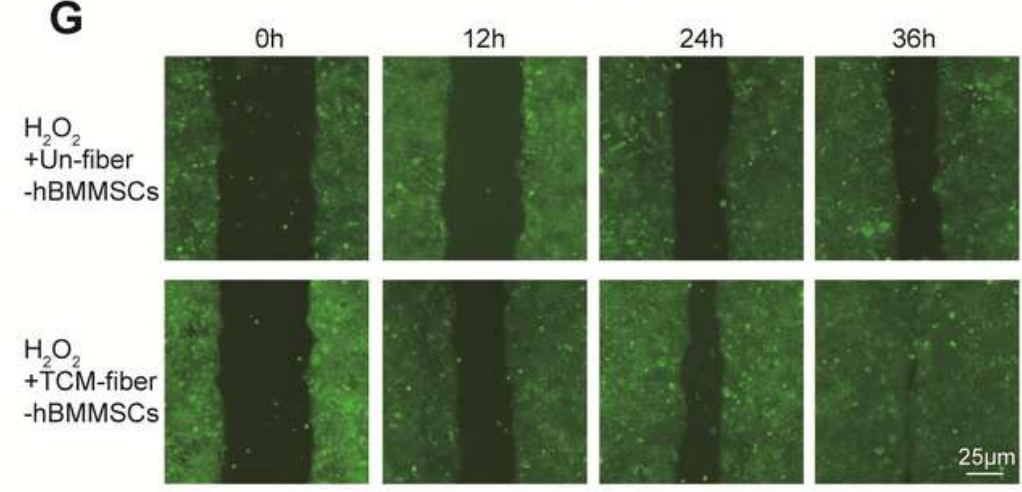

$25 \mu \mathrm{m}$
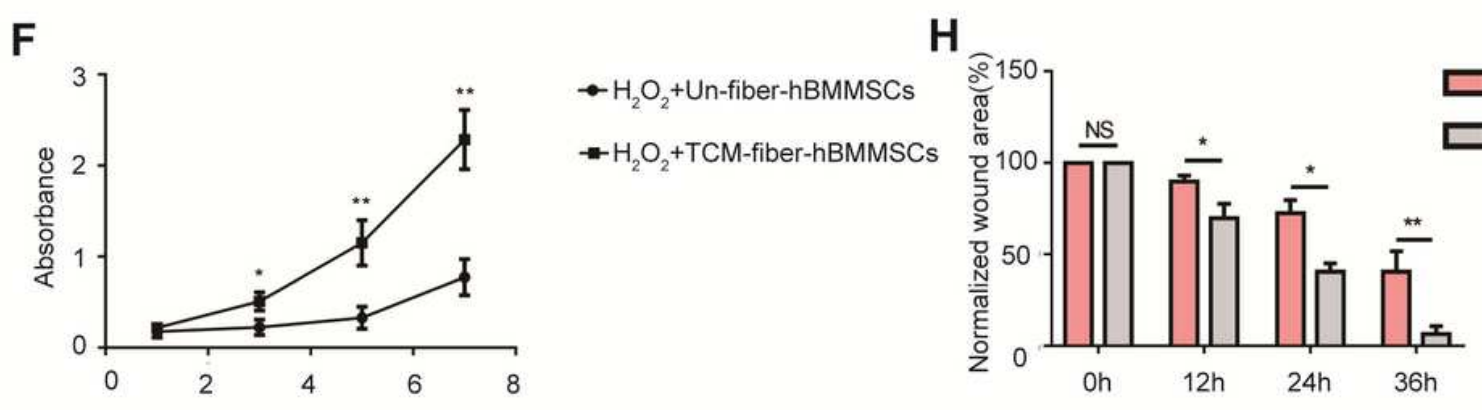

$\mathrm{H}_{2} \mathrm{O}_{2}+$ Un-fiber-hBMMSCs

$\mathrm{H}_{2} \mathrm{O}_{2}+\mathrm{TCM}$-fiber-hBMMSCs

$-\mathrm{H}_{2} \mathrm{O}_{2}+\mathrm{TCM}$-fiber-hBMMSCs

\section{Figure 7}

Physiochemical and biological characterization of TCM-fibers(LPS/IFN- $\gamma$ activated human THP-1 cell membrane modified(lactic-co-glycolic acid) (PLGA) nanofibers). (A). Representative fluorescent images of the TCM-fibers and Un-fibers after coumarin and Dil staining(scale bar $=20 \mu \mathrm{m})$. (B). Detection of $\mathrm{Na}+\mathrm{K}+$ ATPase and CD11c on the TCM-fibers and Un-fibers (without LPS/IFN-y activated RAW263.7 cell membrane) by Western blotting. (C). Representative images of the morphology of the TCM-fibers by scanning electron microscopy (scale bar $=10 \mu \mathrm{m}, 5 \mu \mathrm{m})$. (D). Size distribution of the TCM-fibers. (E). Fluorescent images of BMMSCs cultured on Un-fibers or RCM-fibers by CFDA-SE staining(scale bar = $5 \mu \mathrm{m})$. (F). CCK-8 assays for cell proliferation of BMMSCs on day 1, day 3 , day 5 and day 7. (G). The representative images of wound areas covered by HACAT cells at 12, 18, 24 and $36 \mathrm{~h}$ (scale bar $=25 \mu \mathrm{m}$ ). $(\mathrm{H})$. Comparisons of normalized wound areas covered by HACAT cells at different time points. Data were 
represented as mean \pm SD. Differences were analyzed by one-way ANOVA with Tukey's multiple comparison tests. $(n \geq 3) * P<0.05, * * P<0.01$

A

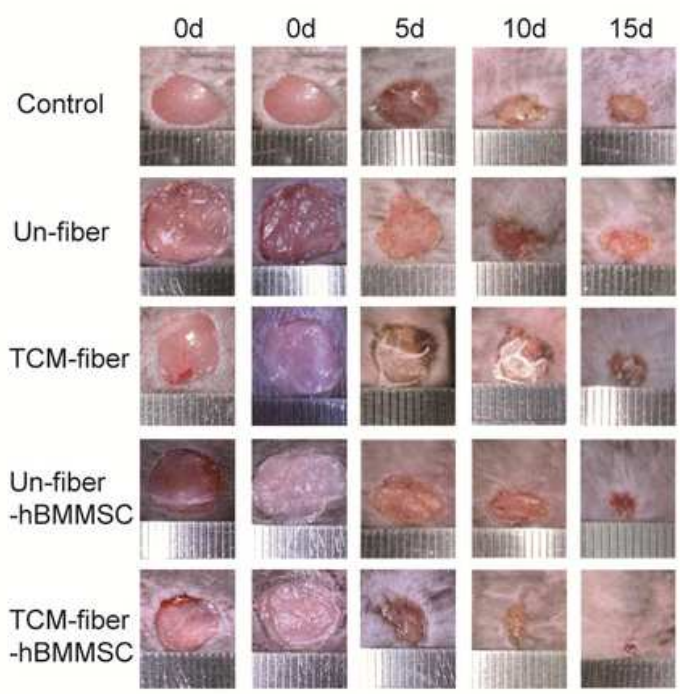

C

Control

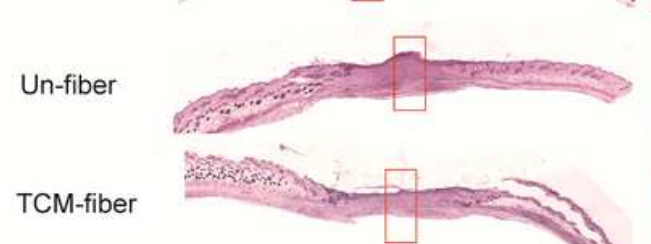

Un-fiber -hBMMSCs

TCM-fiber -hBMMSCs
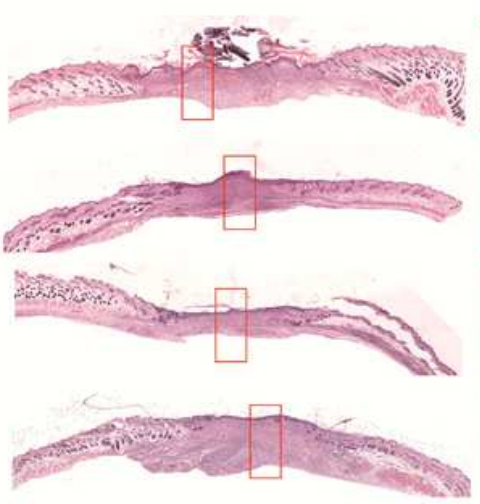

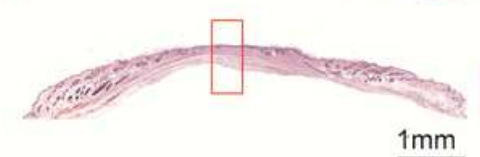

Enidermis Dermis Hvpodermis
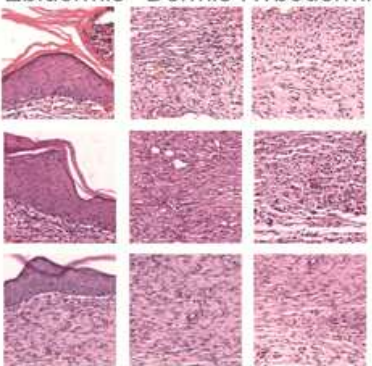

D

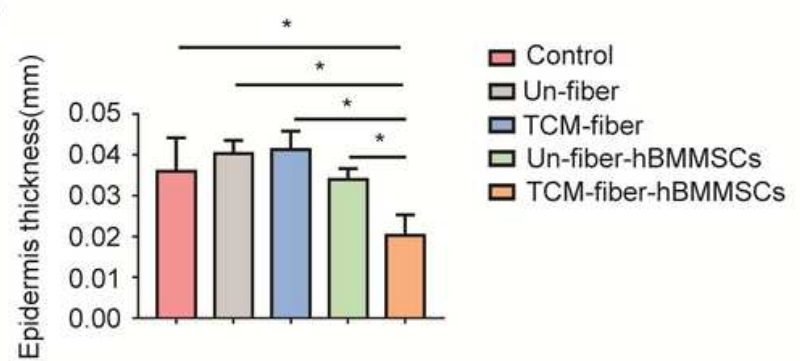

$\square$ Control

$\square$ Un-fiber

$\square$ TCM-fiber

$\square$ Un-fiber-hBMMSCs

$\square$ TCM-fiber-hBMMSCs

$\mathbf{E}$

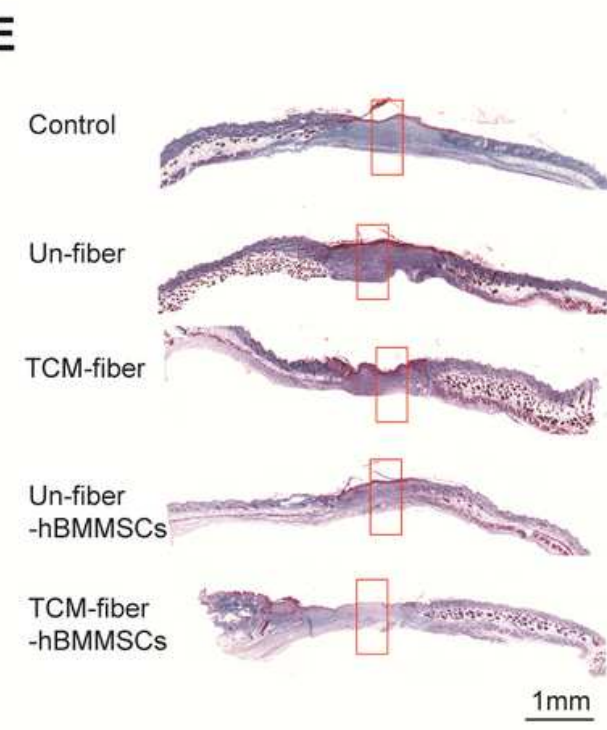

Epidermis Dermis Hypodermis
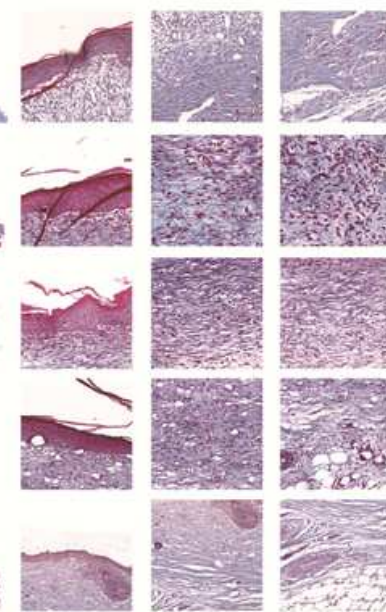
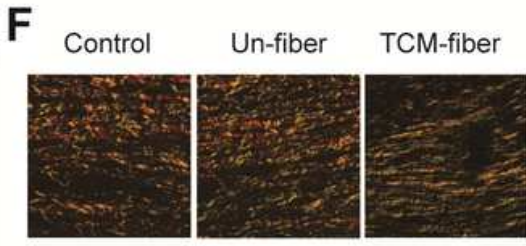

G

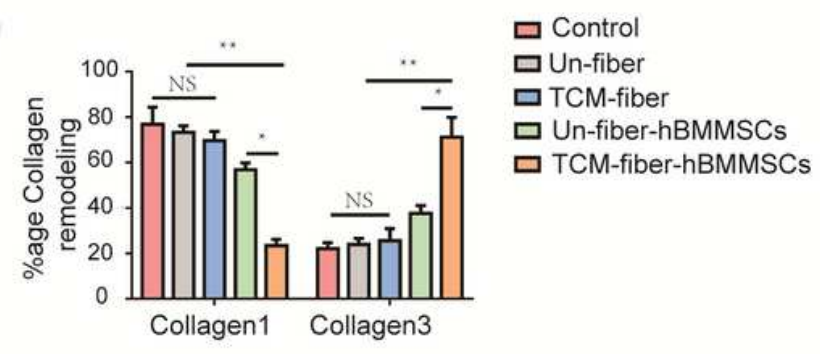

\section{Figure 8}

TCM-fibers-hBMMSCs improve wound healing in diabetic wound. (A). Representative images of the wounds at day 0, 5, 10 and 15 post-wounding, respectively. (B). Quantification of the residual wounds areas at day $0,5,10$ and 15, respectively. (C). Representative images of H\&E staining for the wounds at day 15 (left) with high-resolution images of epidemis, dermis and hypodemis (Right)(scale bar $=1 \mathrm{~mm}$, 
$50 \mu \mathrm{m})$. (D). Quantification of average epidermal thickness of the wounds. (E). Masson's trichrome staining for tissue sections (Left) with high-resolution images of collagen deposition in the epidemis, dermis and hypodemis (Right) in diabetic mouse wounds(scale bar $=1 \mathrm{~mm}, 50 \mu \mathrm{m})$. (F). Picrosirius red staining for collagen deposition and remodelling in the wounds from diabetic mice(scale bar $=50 \mu \mathrm{m})$.

(G). Comparisons of collagen 1 and collagen 3 deposition among different groups. Data were represented as mean \pm SD. Differences were analized through two-way and oneway ANOVA with Tukey's multiple comparison tests. $(n \geq 8)$ NS: non-significant, ${ }^{*} P<0.05, * * P<0.01$

\section{Supplementary Files}

This is a list of supplementary files associated with this preprint. Click to download.

- graphicsummaryimage.jpg

- Supplementarymaterials20220225Shaoying.pdf

- scheme1.tif 